\title{
Reading memory traces in cultured neuronal networks by probabilistic analysis
}

\author{
Dissertation \\ for the award of the degree \\ "Doktor rerum naturalium (Dr. rer. nat.)" \\ GGNB program Physics of Biology and Complex System
}

Georg-August-Universität Göttingen, Physics Faculty

submitted by

Ghazaleh Afshar

from Tehran, Iran

Göttingen 2013 


\section{Member of the Thesis Committee}

- Prof. Dr. Fred Wolf (Reviewer)

Department of Nonlinear Dynamics,

Max Planck Institute for Dynamics and Self-Organization

- Prof. Dr. Walter Stühmer

Department of Molecular Biology of Neuronal Signals, Max Planck Institute for Experimental Medicine

- Prof. Dr. Theo Geisel

Department of Nonlinear Dynamics,

Max Planck Institute for Dynamics and Self-Organization

\section{Additional reviewer}

- Prof. Dr. Florentin Wörgötter

Bernstein Center for Computational Neuroscience

Date of the oral examination: 10th Jan 2014 
I hereby declare that I prepared this doctoral thesis, entitled "Reading memory traces in cultured neuronal networks by probabilistic analysis", on my own and with no other sources and aids than those cited.

Ghazaleh Afshar,

Feb 10th 2014, Göttingen 


\section{Contents}

\begin{tabular}{lll}
\hline 1 & Introduction & 1
\end{tabular}

1.1 Synchronization and Bursting dynamics . . . . . . . . . . 2

1.2 Recording and Stimulation Techniques $\ldots \ldots$. . . . . . . 7

1.3 Leader-Follower . . . . . . . . . . . . . . . . . . . . . . . . . 10

2 Optogenetic stimulation effectively enhances intrinsically gen$\begin{array}{ll}\text { erated network synchrony } & 13\end{array}$

2.1 Introduction . . . . . . . . . . . . . . . . . . . . . . . . . . 13

$2.1 .1 \quad$ Bursting in neuronal networks . . . . . . . . . . . 13

2.1 .2 Network level plasticity . . . . . . . . . . . . . . . . . 14

2.1 .3 Aim of the study . . . . . . . . . . . . . . . 15

2.2 Results . . . . . . . . . . . . . . . . . . . . . . . . . . . 17

$2.2 .1 \quad$ Experimental system design . . . . . . . . . . . 17

2.2 .2 PSTH - Network response to the light stimulation . . . 18

$2.2 .3 \quad$ Network firing rate increases after stimulation . . . . . 21

$2.2 .4 \quad$ Network bursting dynamics changes after stimulation . 24

$2.2 .5 \quad$ Interneuronal spike correlations increase after stimulation 26

$2.2 .6 \quad$ Optogenetic modification of network dynamics is NMDAand AMPA- receptor dependent . . . . . . . . . . . . 27

2.3 Discussion $\ldots \ldots \ldots \ldots \ldots$ 
3 Enhancing burst activation and propagation in cultured neuronal networks by photo-stimulation 35

3.1 Introduction . . . . . . . . . . . . . . . . . . . 35

3.1 .1 Leaders and Followers . . . . . . . . . . . . . . 36

3.1 .2 Aim of the study . . . . . . . . . . . . . 38

3.2 Results . . . . . . . . . . . . . . . . . . . . . . . 38

3.2.1 Changes in the IBFR maximum peak after photo-stimulation 38

3.2.2 Changes in the IBFR maximum peak delay after photostimulation . . . . . . . . . . . . . 42

3.3 Discussion . . . . . . . . . . . . . . . . . . 45

$\begin{array}{lll}4 & \text { Materials and methods } & 48\end{array}$

$4.1 \quad$ Experimental Preparation . . . . . . . . . . . . . . . . . . . 48

4.1.1 Cell culture, transduction and multi-electrode array recordings . . . . . . . . . . . . . . . 48

4.1 .2 Whole field blue light stimulation . . . . . . . . . . . . 49

4.1 .3 Control experiments . . . . . . . . . . . . . . 50

4.1 .4 Pharmacological experiments . . . . . . . . . . 50

4.2 Network Dynamics Data Analysis . . . . . . . . . . . . . . 50

$4.2 .1 \quad$ Active Electrode (AE) Identification . . . . . . . . . 50

$4.2 .2 \quad$ Mean Firing Rate of AEs. . . . . . . . . . . . . . . . . 51

4.2 .3 Peri-stimulus time histogram . . . . . . . . . . . . . 51

4.2 .4 Burst Detection . . . . . . . . . . . . . . 51

4.2 .5 Cross Correlation . . . . . . . . . . . . . . . 55

4.3 Leaders and Followers $\ldots \ldots \ldots$

4.3 .1 Leader Detection . . . . . . . . . . . . . . 55

4.3 .2 Intra-burst firing rate (IBFR) $\ldots \ldots \ldots$. . . . 57

4.3 .3 Peak Delay $\ldots \ldots \ldots$ 
$\begin{array}{lll}5 & \text { General discussion } & 58\end{array}$

\begin{tabular}{ll}
\hline Bibliography & 63
\end{tabular} 


\section{Chapter 1}

\section{Introduction}

The brain can be regarded as a highly interacting complex system exhibiting emergent phenomena such as learning and memory. Studying its functions requires reducing its complexity to basic units. The basic unit of brain is of course a single neuron. But in order to understand the interesting dynamics of interconnected neuronal systems such as learning, synchronization and plasticity, one need simple connected neuronal networks like neuronal cultures. The connectivity of the cultured networks in-vitro is of course very different from in-vivo structure. However, in case of in-vitro cultures, the structure-function relations are indeed easier to measure and to express in the context of simplified connectivity patterns. Moreover, such relations could then be generalized to help understanding activity in the complex and realistic in-vivo structures. The history of cell culturing goes back to the beginning of the 20th century, when pioneering work of Harrison showed that cells and neurons can be maintained alive outside of the animal. By reviewing the origins and progress of neuron culture methods, beginning with Harrison's pivotal publication, we can appreciate how acquiring, improving, and interjecting new methodologies into conventional practices enhances our ability to advance understanding of neuronal structure and function. The currently accepted protocol for culturing primary cultures of neurons is well defined. Cultures are typically prepared from neurons of specific regions of the brain, such as the hippocampus or cortex, dissociated, and plated over 
glass. The culturing process is reproducible and versatile enough to permit the study of neural cultures in a variety of geometries or configurations, and with a broad spectrum of experimental tools. Modern techniques permit to maintain neural cultures healthy for several months, making them excellent model systems to study development (Wagenaar et al., 2006a; Marom and Shahaf, 2002), learning (Shahaf and Marom, 2001; Marom and Shahaf, 2002; Eytan et al., 2003) and plasticity (Maeda et al., 1998; Jimbo et al., 1999).

One of the well stablished methods of extracellular recording, originated with the work of Gross (Gross, 1979; Gross et al., 1993), is the Multi-electrode array (MEA) technology. MEA recording allows monitoring neuronal networks activity with dozens of electrodes generating huge amounts of data. Experiments combining multi-electrode array technology and optical neurostimulation using Channelrhodopsin-2 offer a very promising way to study neuronal network. It is known that in-vivo neural systems are able to adapt selectively to stimuli with different features (Eytan et al., 2003); analogous stimulationdependent dynamics are likely to develop in our in-vitro cultures. Using the aforementioned techniques, we could monitor the network-level plasticity in cultured neuronal networks.

\subsection{Synchronization and Bursting dynamics}

Synchronized network activity is ubiquitous across the brain. It has been observed in many brain areas such as visual cortex (Martinez-Conde et al., 2000), lateral geniculate nucleus (Reinagel et al., 1999) and hippocampus (Leinekugel et al., 2002). The brain generates extensive spontaneous network synchronized activity, in the absence of extrinsic input, exhibiting different rhythms of oscillation (Buzsáki, 2006). If the synchronization measured during ongoing, spontaneous activity becomes abnormal, it can indicate dysfunction of the underlying cortical network. For example, excessive synchrony occurs during epileptic seizures (Holtkamp et al., 2011) and Parkinson's disease (Uhlhaas and Singer, 2006). On the other hand, abnormally weak synchrony has been associated with disorders such as schizophrenia (Uhlhaas and Singer, 
2010) and autism (Yizhar et al., 2011). Thus, a healthy cortical network must be regulated such that spontaneous synchrony avoids pathological extremes and remains moderate on average.

Neuronal cultures in-vitro, in the absence of external stimuli, display collective bursting activity similar to that seen in-vivo. Bursting appears to be a typical behavior for a random neural network and is observed in a large variety of preparations, including cultures from dissociated hippocampal neurons (Cohen et al, 2008; Mazzoni et al., 2007) and cortical neurons (Wagenaar et al., 2006a; Yang et al., 2012; Maeda et al., 1995). Initially, a slow rhythmic bursting activity has been described in the developing hippocampus and later found in other brain areas, and this activity is assumed to play a major role in the developmental organization of these structures (Bacci et al., 1999; Crépel et al., 2007; Goodman and Shatz, 1993). Rhythm activity is also present in the isolated hippocampus, indicating that it is generated intrinsically; however the rules governing its generation are still elusive. Since it is hard to control the parameters that shape the network activity in-vivo, cultured networks of neurons in-vitro are studied as a simplified model system for the governing rules of emergence, generation and spread of spontaneous synchronized network bursting and also to understand how these activities can be modified.

The seeded cells in a neuronal culture first grow dendritic and axonal processes after few days in-vitro and continue until reaching a maturation level to elaborate these processes. During this maturation process, chemical synaptic connections are established and spontaneous network dynamics develop in the culture. The neuronal network in general can have three different form of firing patterns (Eckmann et al., 2007): 1) Asynchronous firing, which typically occurs at very early developmental stages (Cohen et al., 2008). 2) Network bursts, which is the most common pattern of activity, which consists of intense activity of bursts evoked as a sequences of spikes with short interspike intervals (ISI) separated by periods of near-quiescence (called interburst-interval). 3) Seizure-like activity, which is an epileptic-like activity, characterized by very long (tens of seconds) episodes of intense synchronized 
firing, that are not observed in neuronal cultures under standard growth conditions (Raghavan et al., 2012).

As previously mentioned, most neuronal networks, even in the absence of external stimuli, produce spontaneous synchronized bursts, however, the mechanistic origin of burst (i) generation (activation, recruitment), (ii) propagation and (iii) termination remain poorly understood. The simple geometry and controlled experiments of in-vitro cultures allow detailed study of the burst dynamics experimentally. A combination of detailed analytical, numerical and statistical analysis and modeling with empirical observations is expected to improve the understanding of burst dynamics and identify strategies how to manipulate and modify bursting dynamics.

Burst generation - While it is observed that synchronized network bursts are a characteristic electrical behavior of neuronal cultures in-vitro, main aspects relevant to understanding of the mechanism of generation of these patterns remain unsolved. Different synaptic and membrane properties contribute to the shaping of synchronized network bursts (Bacci et al., 1999; Cohen and Segal, 2009). Network activity requires both excitatory and inhibitory neurons. Bursts in cultured hippocampal networks critically depend on excitatory glutamatergic neurotransmission via NMDA receptors (Cohen et al., 2008) and AMPA receptors (Arai and Lynch, 1998). In addition, GABAergic interneurons play an important role in the synchronization of network activity and in the timing of the individual bursts (Cohen et al., 2008).

Burst propagation - It has been shown that with maturation of the network, the frequency and propagation velocity of bursts increase markedly in cultures (cortical cultures (Maeda et al., 1995), hippocampal cultures (Cohen et al., 2008)). The ignition and propagation mechanism of bursts have been studied in 1-D neuronal networks, which provide a simple system to compare models of collective dynamics and the actual measured behavior of neural cultures (Feinerman et al., 2005 \& 2007; Feinerman and Moses, 2006). In 2-D neuronal cultures, the dynamics is markedly more complex compared to 1-D cultures. The observations in 1-D cultures indicate that the firing patterns are generated in one of several burst initiation zones (BIZ) (Feinerman 
et al., 2007). From these BIZs, activity propagates as a wave through the line. On the other hand, in 2-D cultures, a select group of neurons, termed as leader neurons, begin to fire ahead of the rest of the network similar to BIZs in 1-D. In this case, the bursts are initiated mostly via one of the leader neurons and then they recruit the follower neurons and propagate as a sequence of spikes distributed over a set of leaders and followers (Ham et al., 2008).

Burst termination - Another interesting aspects of bursts dynamics is how the duration of network bursts are determined or in other words which mechanism cause the termination of burst. Yet the exact mechanism that determine the duration of synchronized burst is not fully understood. Recent evidence suggests that this may depend on the combination of release probability and replenishment of readily releasable vesicle pools (Jones et al. 2007). However, it can also be likely to be due to other factors like postsynaptic parameters such as glutamate receptor desensitization (Arai and Lynch, 1998) or strong afterhyperpolarization (AHP) (Darbon et al., 2002). Recent study by Cohen and Segal (2011), argued that the factor limiting burst duration is most likely the exhaustion of vesicle pools in the presynaptic terminals.

Plasticity and learning - The existence of techniques to maintain neural cultures healthy for several months and being able to reproduce similar cultures, makes neuronal cultures a promising model systems for studying problems such as plasticity (Maeda et al., 1998; Jimbo et al., 1999) and learning (Marom and Shahaf, 2002; Shahaf and Marom, 2001; Eytan et al., 2003). Using neuronal cultures, one can study learning in the sense of a persistent change in the way neuronal activity reacts to a certain external stimulus. It is known that the neuronal coupling is often dynamic, and changes according to the activity of the coupled systems. The correlation between synchronized firing and changes in coupling strengths is known as Hebb's rule (Hebb, 1949). According to Hebbian Rule, synapses increase their efficiency if the synapse persistently takes part in firing the postsynaptic target neuron. This change in coupling is called "neuronal plasticity", which happens specifically due to the activity-dependent modification of the strength 
or efficacy of synaptic transmission at preexisting synapses. Synaptic plasticity can be either enhanced or depressed by activity, and these changes span time scales ranging from millisecond to hours, days, and presumably even longer. Synaptic plasticity can be divided into three broad categories (Abbott and Regehr, 2004): (1) long-term plasticity, involving changes that last for hours or longer, and it is thought to underpin learning and memory (Brown and Milner, 2003; Lynch, 2004); (2) homeostatic plasticity, which allows neural circuits to maintain appropriate levels of excitability and connectivity (Turrigiano \& Nelson, 2004); (3) short-term plasticity, which occurs over milliseconds to minutes (Zucker and Regehr, 2002) and allows synapses to perform computational functions in neural circuits. Here, we aim to study learning and memory, therefore, we are more interested in long-term plasticity. Long-term plasticity can be separated into two types of phenomena: (1) long-term potentiation "LTP", which represents the enhancement of synaptic transmission; or, (2) long-term depression "LTD, which represents weakening of synaptic transmission. Experimentally, LTP and LTD can be induced using different methods (Zucker and Regehr, 2002; Dan and Poo, 2006; Lynch, 2004). One of these methods is spike timing dependent plasticity. In 1997, Markram et al. controlled pre- and postsynaptic spike timing using dual whole-cell recording, and discovered that the sign and magnitude of LTP and LTD indeed depended on the order and timing of pre- and postsynaptic spikes on the $10 \mathrm{~ms}$ time scale. This dependence was characterized in detail by Bi and Poo (1998) and named "spike-timing-dependent plasticity" (STDP) by Abbott and Nelson (2000). In STDP, LTP occurs when presynaptic spikes lead postsynaptic spikes up to $\sim 20 \mathrm{~ms}$, and LTD occurs when postsynaptic spikes lead presynaptic spikes by up to 20-100 ms (Bi and Poo, 1998; Markram et al., 1997). Spike time dependent plasticity requires multiple (typically 60-100) pre-post spike pairs. This is termed "Hebbian" STDP because it strengthens synaptic inputs that lead postsynaptic firing and depresses input that are uncorrelated with postsynaptic spikes. 


\subsection{Recording and Stimulation Techniques}

To study the dynamics and to manipulate bursting dynamics in large population of neurons, at the network level, we needed to develop a technology for stimulating the network while simultaneously recording the ongoing spontaneous activity. In recent years, several pioneering studies and technological development have contributed to the emergence of the novel field of network electrophysiology. Traditionally, intracellular recordings using patch-clamp technique (Neher, 1992) provided information about the activity of only one or a pair of neurons. Although this technique has the advantage of allowing to carry out accurate measurements of voltage changes in the neurons, but given the sophistication of the equipment that patch-clamp requires, measurement of substantially larger number of neurons are not feasible yet. Calcium imaging using fluorescent dyes is another technique (Gee et al., 2000). This technique has a high spatial resolution which allows monitoring the whole neuronal culture, however, it has a low temporal resolution and moreover because it affects the neurons chemically, after few hours of measurement (typically 4-6) they will slowly lose their activity and will eventually die. With the advent of improved Multi-electrode Array (MEA) technologies, the number of simultaneously recorded channels has been increased to previously inaccessible ranges. MEAs allow the detailed monitoring of the activity of hundreds of neurons. The pioneering work of Gross (Gross, 1979; Gross et al., 1993), founded a sophisticated and commercially available technology with ready-made electrode arrays of different sizes and full electronic access and amplification equipment (Potter et al., 2006). MEAs allow the monitoring of the activity of hundreds of neurons. The advantage of the existing MEAs is to provide precise measurements of the electric signal, fast responses, and high temporal resolution. Therefore this technique is extensively used recently to study different problems such as studying learning and memory (Wagenaar et al., 2006a; Shahaf and Marom, 2001; Eytan et al., 2003; Maeda et al. 1998; Jimbo et al., 1999). Randomly grown cultures on MEAs, have the disadvantage that some electrodes may not cover much activity and some may record the activity from several neurons which may 
need some spike sorting to separate the activity from the different neurons. This issue can be solved by new developing techniques of pattern culturing which makes neurons to grow only in the vicinity of the electrodes (Nam et al., 2004).

After choosing a proper recording technique, one has to choose the proper stimulation technique to let simultaneous recording and stimulation. Electrical stimulation can be used for collective stimulation combined with calcium imaging technique for recording (Reiher et al., 2005), which has the disadvantage of low temporal resolution. There has been also techniques to use electrical stimulation combined with MEAs to have local stimulation (Eytan et al., 2003), but this technique suffers from several known disadvantages, e.g. substantial artifacts for MEA recordings, difficulty to localize multi-spot stimulation and interference between the multi electrode stimulation and recording loops (Wagenaar and Potter, 2002). Chemical inductions, on the other hand, requires chronic treatment with pharmacological agents that might interfere with the physiological state of the neurons and offers no temporal control (Molnár, 2011). The alternative to the aforementioned stimulation techniques is optogenetic stimulation. Optogenetic stimulation generates almost no artifacts and allows specific and global stimulation with high temporal resolution. In this technique, genes coding for special light-sensitive proteins are delivered to the neuronal cells by transfection. These light-sensitive proteins can be naturally occurring or they can be chemically modified to become photosensitive. The first naturally occurring proteins used to control neuronal activity were Channelrhodopsins. Channelrhodopsins are the primary photoreceptors in the eyespot of the green alga C. Reinhardtii that are responsible for phototactic and photophobic responses. There are two types of Channelrhodopsin in C. Reinhardtii, one with fast kinetics and poor light sensitivity (Channelrhodopsin-1, which is mainly responsible for photophobic and phototactic responses) (Nagel et al., 2002) and Channelrhodopsin-2 which has a slower kinetics and higher sensitivity (Nagel et al., 2003). Channelrhodopsin-2 is a light-activated non-selective cation channel (Nagel et al., 2003) that is widely used in the field of neuroscience recently (Boyden et al., 2005). Illumination of Channelrhodopsin-2 
expressing cells with blue light $(475 \mathrm{~nm})$ induces a sustained inward current which depolarizes the neuron and can trigger spikes. Stimulating such systems can be performed for extended periods of time (Boyden et al., 2005). Several variants of Channelrhodopsin-2 have been developed, e.g. ChETA mutants were engineered as faster Channelrhodopsin2 variants, which can be used to drive spiking in neurons at frequencies greater than $40 \mathrm{~Hz}$ (Gunaydin et al., 2010). The area of stimulation can be precisely determined by confined illumination. The progress in optical technologies has a great impact on designing and controlling optical stimulation with a very high spatio-temporal resolution, for example by using Digital Micromirror Devices (DMD) to selectively illuminate a subset of cells with arbitrary dynamic user-controlled patterns of light (Wang et al., 2007).

Burst detection - Generally, the spontaneous activity emerging from cultured neuronal networks ranges from asynchronized spiking between 3 and 7 days in-vitro to more organized synchronized bursting over the following week (Cohen et al., 2008). Eventually, after maturation of the culture (from the third week in-vitro), these networks exhibit a complex non-periodic, synchronized bursting activity. Qualitatively, bursts can have different shapes, sizes ad durations distributions (Marom and Shahaf, 2002; Wagenaar et al., 2006a). At the level of a single neuron, bursts are defined as a fast sequence of spikes with a very short inter-spike interval (ISI) and separated by a relatively longer interval called inter-burst interval. Therefore, the ISI histogram of burst activity is bimodal. The first mode with short ISIs results from spikes induced within the bursts and the second mode with larger ISI corresponds to the inter-burst intervals. When the entire network gets involved in the burst activity at the same time, the phenomena is described as network burst. Synchronized network bursts consist of sequences of synchronized bursts, which spread across all or part of the culture. The burst structure and dynamic can be used to study the characteristic behaviors of neuronal cultures. Thus, an accurate detection of bursts and synchronized network bursts is a necessary starting point. Depending on type of recording, one can use different methods of burst detection. In this thesis, I am using MEA and therefore we focus on burst detection methods using this extra- 
cellular technique of recording. Most of the proposed methods are largely user-dependent and require the setting of several parameters. The most important parameter to be set is the ISI threshold, which determines which spikes to be considered within bursts or outside of bursts. Using only a fix ISI threshold can cause the problem of excluding or including some spikes within the bursts by mistake. In order to fix this problem, some groups are considering also a threshold crossing by analyzing the temporal development of population firing rate (Eckmann et al., 2008) or by using a larger time window to include the slightly less closely spaced spikes which are definitely also not in the inter-burst interval and a minimum number of spikes within a burst (Wagenaar et al., 2005a). There are also proposed self-adapting methods capable of detecting changes in the bursts' and network bursts' features for different experimental conditions (Pasquale et al., 2010). However, in the aforementioned method also, the maximum ISI allowed for spikes within a burst and minimum number of consecutive spikes belonging to a burst were fixed, which means they can not be fully automated. Therefore, as previously mentioned, the burst detection algorithms are user-dependent. In this study, I have modified the method proposed by Wagenaar et al. (2005a) and the detailed burst detection algorithm is explained in Chapter 4.

\subsection{Leader-Follower}

In cultured neuronal networks exhibiting synchronized burst activity, the network repeatedly goes from bursting to a quiescent state. The process of return to the bursting state is stochastic and depends on sufficient random synaptic activity igniting a positive feedback loop that initiates the next collective network burst (Menendez de la Prida et al., 2006). An important question is: What triggers the generation and spread of network synchronized activity? Two different aspects are being discussed. In one, it is suggested that a network burst is generated once sub-threshold synaptic events in a small population of neurons cross threshold to produce a massive burst (Feinerman et al., 2005). The other hypothesis proposes that leader 
neurons generate the activity which is spread to follower ones (Ham et al., 2008). In this study, the second hypothesis is considered.

There are numerous studies using quantitative analysis to study ignition and spread of collective spontaneous electrophysiological activity in cultures (Ham et al., 2008; Eytan and Marom, 2006; Eckmann et al., 2008; Feinerman et al., $2005 \& 2007)$. It has been shown that the order of activation within a synchronized burst is non-random but rather hierarchical (Eytan and Marom, 2006). Previous theoretical and experimental research showed that multiple ignition sites, termed as initiation zones (Feinerman et al., 2005 \& 2007), privileged neurons (Eytan and Marom 2006), leader neurons (Eckmann et al., 2008), or major burst leaders (MBL) (Ham et al., 2008), create network bursts by recruiting further neurons. Leader neurons are relatively robust and they carry information about the identity of the burst and they are supposed to be part of an underlying sub-network that is excited first (Eckmann et al., 2008), which then involves the follower neurons into the orchestrated activation of neural cell assemblies. Leader-follower neuron relationship should reflect the dynamical state of the network and might be used to infer network-level changes. Also, the temporal relationships between leader and follower neurons can be used to reconstruct network topology (Ham et al., 2008).

In this thesis, I am studying the enhancement of the collective burst dynamics, burst activation and propagation in hippocampal neuronal networks in-vitro by using optogenetic techniques. Channelrhodopsin-2 transfected hippocampal neuronal cultures were grown on MEAs for simultaneous optical stimulation and electrical recording from neuronal networks. We use cultured networks consisting of dissociated hippocampal neurons from embryonic rats (E18), plated on poly-D-lysine/laminin coated multi-electrode arrays. At the third day after the in-vitro culture, the cells began to grow dendritic and axonal processes. This growth process continued till day 21, when synaptic maturation is achieved. In order to use optical stimulation, the hippocampal neuronal cultures were transfected with Channelrhodopsin2 at 14 DIV with AAV-CAG-CHOP2 virus. The cell culture preparations 
and recordings are done by Dr. A. El Hady at Max Planck Institute for Experimental Medicine, in the lab of Prof. Stühmer. Upon maturation, these networks develop an electrical activity that is characterized by synchronized collective bursts (Wagenaar et al., 2006a). In Chapter 2, I study the network level plasticity induction using optical stimulation. We found low frequency photo-stimulation protocols that are sufficient to induce potentiation of network bursting, modifying bursting dynamics and increasing interneuronal synchronization. This study shows that mild stimulation protocols that do not enforce particular activity patterns onto the network can be highly effective inducers of network-level plasticity. In Chapter 3, I investigate how this network level plasticity can be indicated in the burst activation and propagation dynamics by looking at the leader-follower neuronal relationship. We found that low frequency photo-stimulation is able to modify the temporal relationship between follower and leader neurons. It is shown that, by using fade-in photo-stimulation, there is a significant shortening of intra-burst firing rate peak delay of follower electrodes after offset of the stimulation compared to unperturbed spontaneous activity. Our study indicated that network level potentiation is associated with a tighter temporal relationship between leader and follower neurons. 


\section{Chapter 2}

\section{Optogenetic stimulation effectively enhances intrinsically generated network synchrony}

\subsection{Introduction}

\subsubsection{Bursting in neuronal networks}

Regular bursting and highly synchronized bursting in-vivo have been observed in hippocampus (Kandel and Spencer, 1961), visual cortex (MartinezConde et al., 2000) and lateral geniculate nucleus (Reinagel et al., 1999). Bursting has been implicated in the development of neural circuits in visual system (Rochefort at al., 2009), in barrel cortex (Minlebaev et al., 2009) and in hippocampus (Leinekugel et al., 2002). Bursting has also been proposed as a coding scheme (Kepecs and Lisman, 2000) for neuronal communication in primary sensory neurons (Krahe and Gabbiani, 2004; Bobkov et al., 2012) and thalamic nucleus (Lesica and Stanley, 2004).

In vitro pyramidal neuron bursting underlies population synchrony in hippocampal and cortical slices (Silva et al., 1991; Miles at al., 1988; Van Drongelen et al., 2003). In addition, neuronal network bursting and synchro- 
nization have clinical implications. Increased neuronal bursting and synchronization are hallmarks for many neurological diseases especially epilepsy (Holtkamp et al., 2011) and Parkinson's disease (Heimer et al., 2006; Uhlhaas and Singer 2006). On the other hand, there are also diseases where a lack of neural synchrony affects cognitive function as has been argued in the case of schizophrenia (Uhlhaas and Singer, 2010).

Cultured networks of hippocampal neurons exhibit spontaneous synchronized network bursts and are well suited as a simplified model system for studying the origins and determinants of bursting dynamics (Mazzoni et al., 2007). Bursts in cultured hippocampal neuronal networks critically depend on excitatory glutamatergic neurotransmission (Cohen and Segal, 2011). In addition, GABAergic inputs participate in the termination of the bursts without affecting its initial phase (Marom and Shahaf, 2002; Cohen et al., 2008). Cultured hippocampal neurons plated on substrate integrated multi-electrode arrays allow recording how large sets of neurons participate in the synchronized network bursting (Wagenaar et al., 2006a).

\subsubsection{Network level plasticity}

To study and manipulate impact of bursting phenomena in large populations of neurons, it is thus crucial to understand how network bursting can be experimentally and therapeutically modified. Optogenetics with its ability to interface with large neuronal populations holds great promise for such applications. Some studies have successfully used optogenetics to mimic natural neuronal synchronization in the olfactory system (Blumhagen et al., 2011) or to manipulate neural synchrony by affecting neuronal spike timing to study its role in neural computation (Han et al., 2009). A study by Tonnesen et al. (2009) has established that optogenetic hyperpolarization of neurons in hippocampal neurons can suppress synchronized epilepticform activity. No studies, however, have used optogenetics to enhance or diminish a network intrinsic ability to generate synchronization. A fundamental question in this respect is whether one can enhance network synchronization using optogenetic stimulation or these network states are stable and cannot be modified. 
Previous studies using electrical stimulation have attempted to modify collective activity within the network (Maeda et al., 1998; Eytan and Marom, 2006; Wagenaar et al., 2006b; Li et al., 2007; Bakkum et al., 2008a,b; Chiappalone et al., 2008; Brewer et al., 2009; Bologna et al., 2010; Ide et al., 2010; le Feber et al., 2010), to induce pathway-specific potentiation and depression after localized stimulation (Jimbo et al., 1999) and to selectively adapt neuronal network for the detection of a specific stimulus (Eytan et al., 2003). Modification of bursting dynamics also appears crucial for the design of novel neurohybrid cultured networks and the establishment of neurocomputing systems (Wagenaar et al., 2005b; Feinerman et al., 2008).

\subsubsection{Aim of the study}

In this study, we used an experimental system combining multi-electrode array recordings and optical stimulation of Channelrhodopsin-2 transducted neurons to study the effect of global activation on synchronized network bursting. We found that whole-field light stimulation of Channelrhodopsin-2 transducted neuronal networks induced a change in the bursting dynamics of the network. In particular, network synchronization increased after light stimulation. These changes persist for long time and reflect the enhanced ability of the network to coordinate the activity of participating neurons. Pharmacological experiments indicate that the changes in bursting dynamics are mediated via excitatory interactions within the network via NMDA and AMPA receptors. Surprisingly, our experiments indicate that slowly fading-in light stimulation, which substantially delays and reduces light driven spiking, was at least as effective in reorganizing network dynamics as much stronger pulsed light stimulation. Our study demonstrates the feasibility to use mild photo-stimulation protocols to increase intrinsic network-level synchronization. It suggests that stimulation protocols that do not enforce particular activity patterns onto the network can be highly effective inducers of network-level plasticity. 


\section{Contributions}

In the following study, I have performed the complete data analysis. This includes implementing burst detection algorithm, burst analysis, firing rate and PSTH analysis. The data was collected by Dr. A. El Hady at the Max Planck Institute for Experimental Medicine, in the lab of Prof. Stühmer. This study is published in a paper by A. El Hady*, G. Afshar*, et al. (2013). 


\subsection{Results}

\subsubsection{Experimental system design}

Our experimental setup (Fig. 2.1) combines multichannel recording using multi-electrode arrays and whole field photo-stimulation. Whole field illumination is performed using a high power blue LED that provides homogeneous illumination of the recorded neurons. Fig. 2.1 shows a 21 DIV embryonic hippocampal neurons plated on 60 channels multi-electrode array (MEA) transduced with an AAV1/2-ChR2-YFP virus (Petreanu et al., 2009; Suska et al., 2013). As has been previously reported, 21 DIV neuronal cultures show spontaneous activity characterized by bursting separated by periods of silence (Wagenaar et al., 2006a; Fig. 2.1). The depicted electrode spike trains in Fig. 2.1 and all our other experiments typically represent multiunit activity as no attempt for spike sorting was made. A typical recording obtained from one culture and the used photo-stimulation protocols are presented in Fig. 2.1. For each experiment, we observed four phases of activity: (1) spontaneous activity of the unperturbed culture; (2) optically driven spiking; (3) a silent period immediately following the termination of light stimulation and (4) spontaneous activity of the culture after stimulus. For each experiment, the spontaneous activity of the culture was recorded for 5 minutes before the onset of the stimulation. Using whole field blue light stimulation, the neuronal cultures were stimulated with either 40 constant amplitude light pulses of 1 second duration or with 40 applications of a light waveform, called fadein, designed as slowly ramping light to the level of the constant pulses over the course of 1 second. Both kinds of stimuli were applied at a frequency of $0.5 \mathrm{~Hz}$. After termination of stimulation the spontaneous activity was recorded for 12 minutes. 

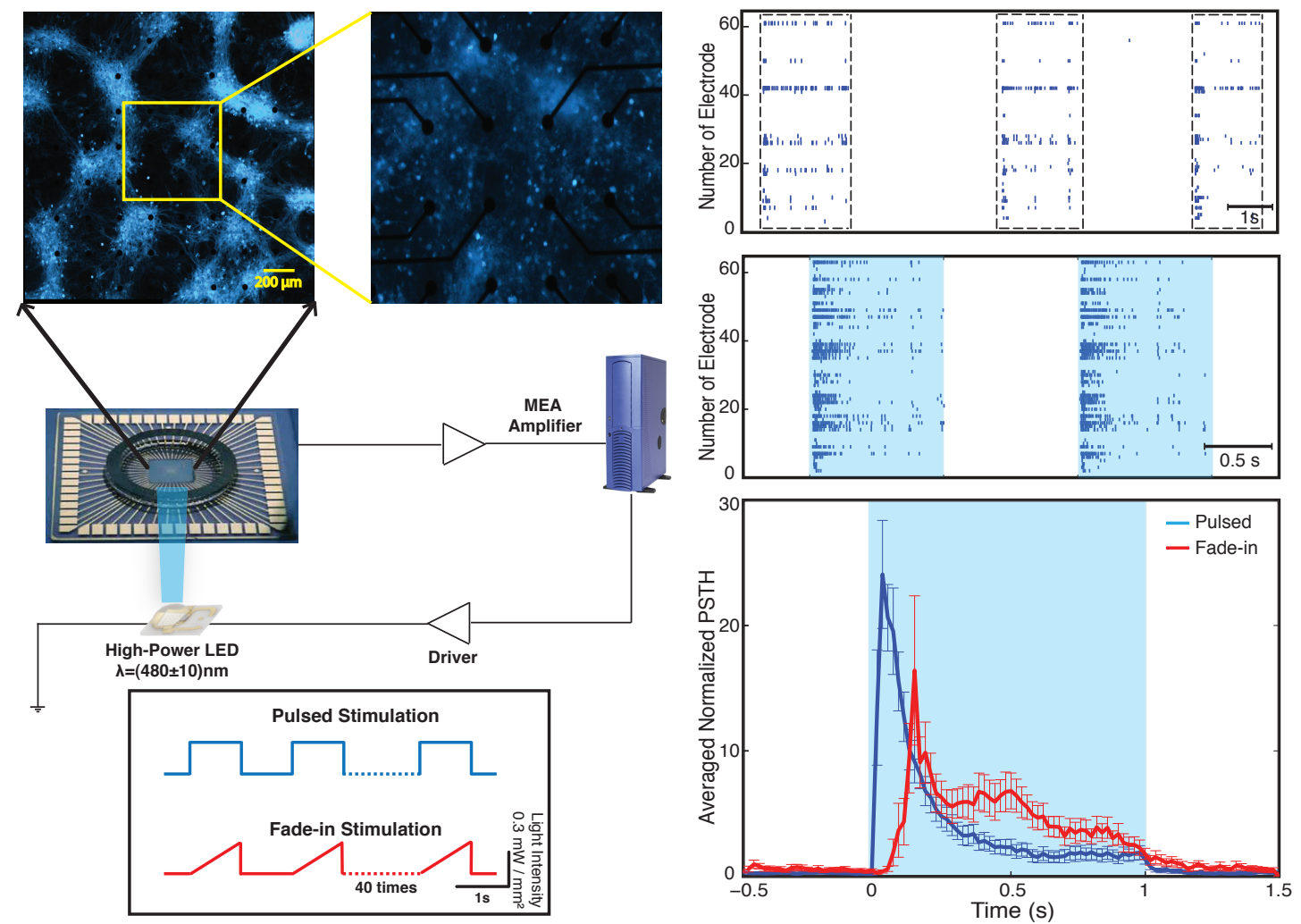

Figure 2.1: Optical Network Electrophysiology. The upper left panel shows the experimental setup including Channelrhodopsin-2 transduced neurons cultured on a multielectrode array stimulated by whole-field blue light illumination using a high-power LED (middle left). The cultures are stimulated with either pulsed or fade-in stimuli (inset lower left). The data is acquired by a MEA amplifier and a recording computer. The upper right panel shows a representative raster plot of spontaneous activity in a network before stimulation across all 60 electrodes. The middle right figure presents evoked activity of the network during pulsed blue-light stimulation. The light blue color marks the duration of blue-light stimulation. The lower right panel present the electrode averaged normalized peri-stimulus time histogram (PSTH) for both pulsed (dark blue) and fade-in stimulation (red).

\subsubsection{PSTH - Network response to the light stimulation}

During the stimulation, the network responded as expected to the blue light stimulation with a phasic increase in the firing rate. The time course of the average firing rate during pulsed stimulation was markedly different from 
that induced by fade-in stimulation. This difference can be seen in the averaged normalized peri-stimulus time histogram (PSTH) plots shown in Fig. 2.1. With pulsed stimulation, the firing rate during each pulse of stimulation rapidly triggered a short latency phasic response. With fade-in photostimulation, the firing rate rose much more slowly and reached a maximum firing rate around 1.5 fold lower than in the case of the pulsed stimulation. In Fig. 2.2 a, b,c,d the averaged normalized firing rate during each pulse of stimulation consequently for pulsed, fade-in, experiments done in the presence of NBQX/Picrotoxin and in the presence of APV/Picrotoxin with pulsed stimulation is shown, which clarifies that there is no significant run-down in evoked responses during each subsequent stimulation in the train of 40 repetition. The average normalized PSTH, which is the average over all normalized firing rate of 40 pulses, is shown in Fig. 2.2 (error bar is the SEM). Moreover, the cumulative distribution of average firing rate during stimulus normalized to average firing rate of the corresponding culture before stimulus (Fig. 2.2 ) shows that there is no significant difference between different stimulation protocols and also in case of experiments with pharmacological blockers there is the same level of evoked activity during stimulus compared to normal experiments without any synaptic blockers. Directly after the offset of stimulation, we observed a silent period that varied in length from a couple of seconds to tens of seconds where no synchronized activity is detected. Afterwards the network resumed the state of ongoing spontaneous bursting activity. 

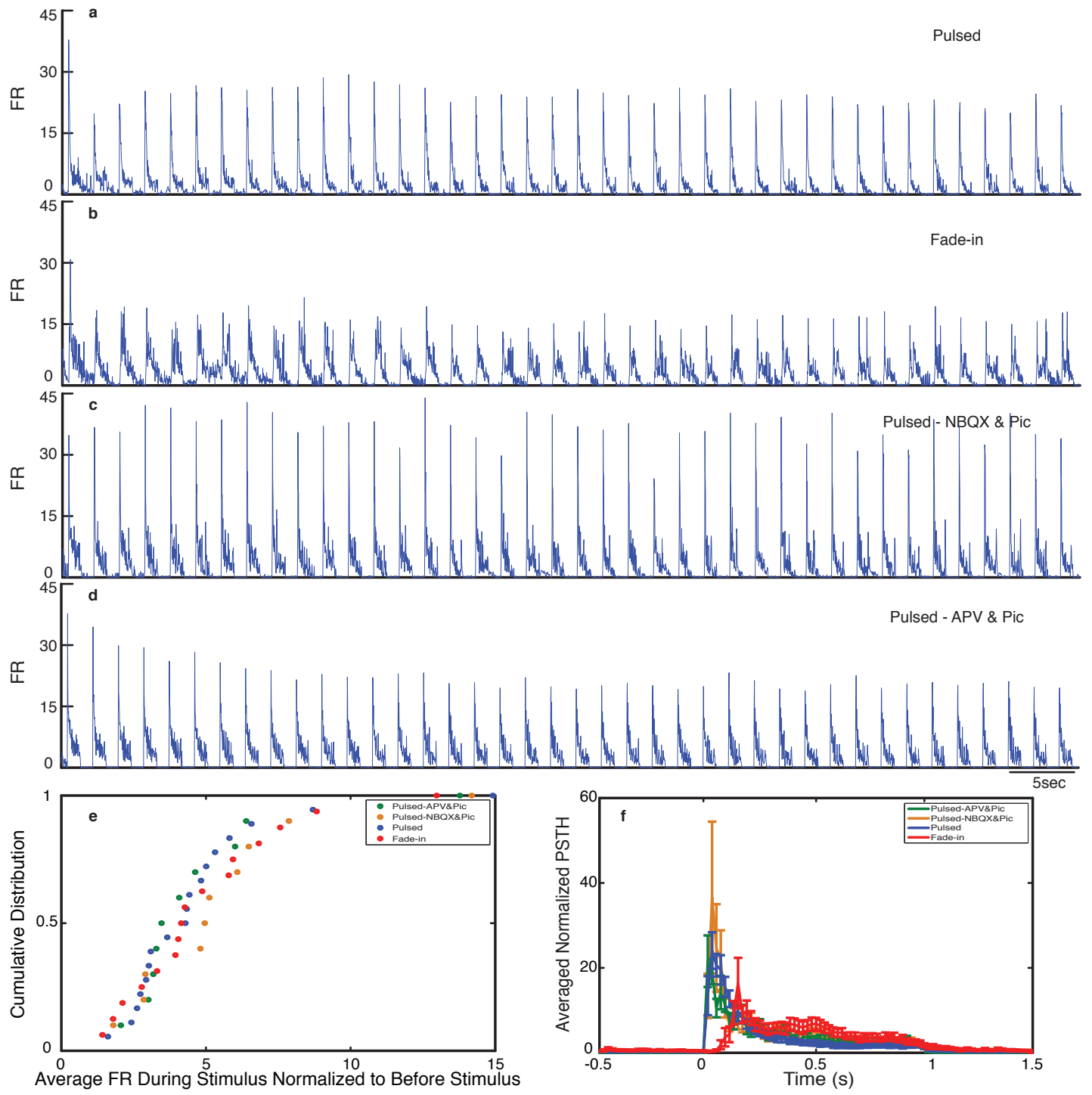

Figure 2.2: Evoked activity during light stimulation. Panels (a,b,c,d) show the average normalized firing rate during each pulse of stimulation (normalized to the average firing rate before stimulus) for pulsed ( $\mathrm{n}=18$ experiments), fade-in ( $\mathrm{n}=16$ experiments), pulsed stimulation on cultures with presence of NBQX/Picrotoxin $(n=10$ experiments) and pulsed stimulation on cultures with presence of APV/Picrotoxin ( $\mathrm{n}=$ 10 experiments). Panel (e) shows the cumulative distribution of average firing rate during stimulus normalized to average firing rate before stimulus. Panel (f) shows the average normalized PSTH over all experiments. 


\subsubsection{Network firing rate increases after stimulation}

We investigated the time course and level of the average firing rate of the network activity after termination of stimulation. With both fade-in and pulsed stimulation, we found that the average normalized firing rate increased significantly after stimulation compared to the unperturbed spontaneous activity prior to stimulation. In case of pulsed illumination (Fig. 2.3 a), the average normalized firing rate $(\mathrm{n}=18$ experiments) substantially increased by $27 \%$ after stimulation ( $p<10^{-7}$, Wilcoxon's rank sum test). As for fade-in stimulation (Fig. 2.3 b), the average normalized firing rate $(\mathrm{n}=16$ experiments) increased by a similar amount of $30 \%$ ( $p<10^{-3}$, Wilcoxon's rank sum test). No significant changes in the average normalized firing rate were found under control conditions either in transduced cultures without light stimulation (n $=7$ experiments) (Fig. 2.4 a) or in non-transduced cultures stimulated with pulsed light stimulation ( $\mathrm{n}=5$ experiments) (Fig. 2.4 b) $(p>0.05$ in both cases, Wilcoxon rank sum test). 

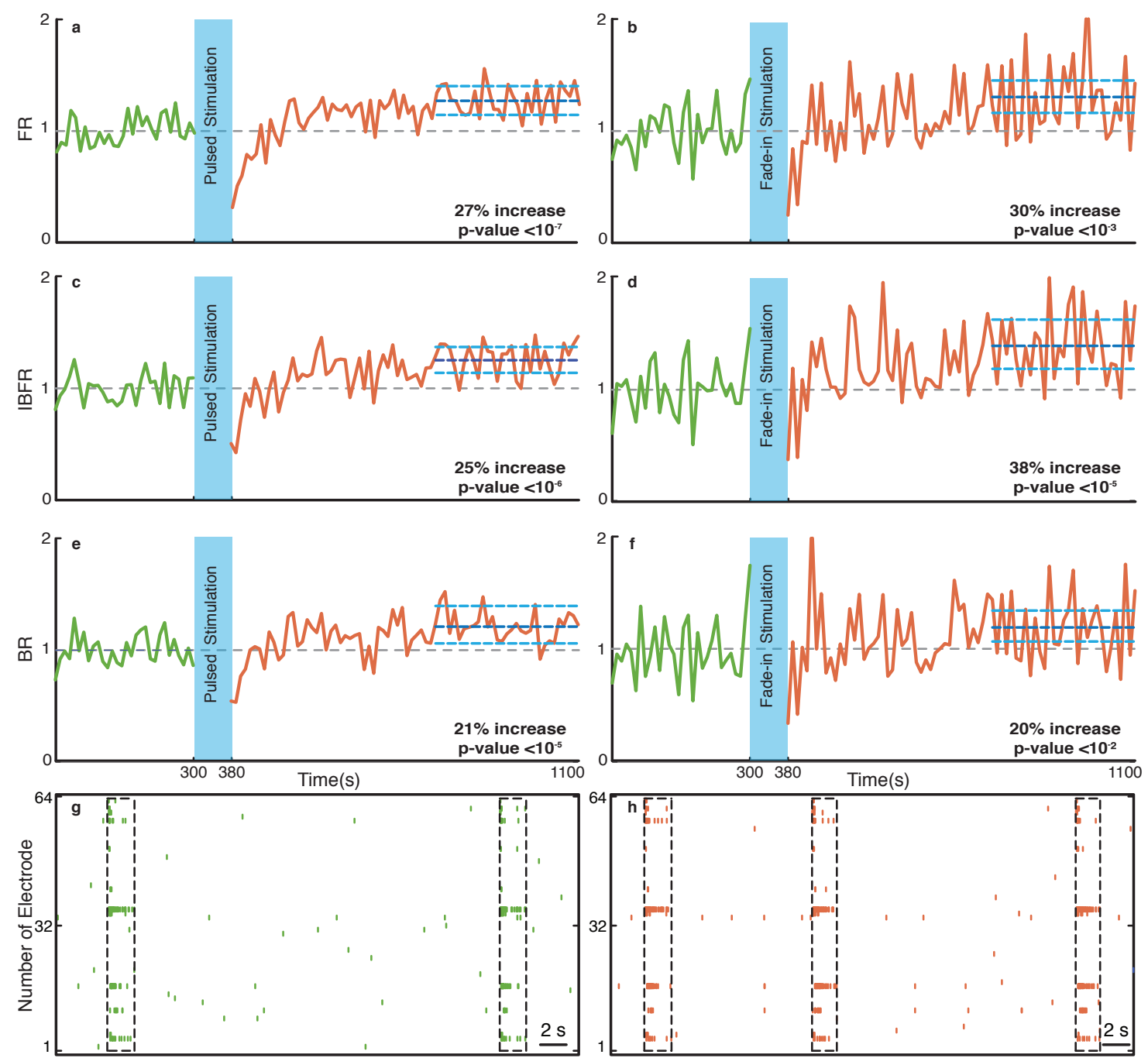

Figure 2.3: Changes in the Network collective dynamics. Plots on the left side are for pulsed stimulation and the plots on the right side are for the fade-in stimulation. $(\mathrm{a}, \mathrm{b})$ Average normalized firing rate before and after stimulation. Here and in the other panels, the dotted grey line marks the mean before stimulation and the dark blue line marks the mean after stimulation. (c,d) Average normalized intra-burst firing rate. (e,f) Average normalized burst rate. $(\mathrm{g}, \mathrm{h})$ An example of spontaneous activity before and after stimulation. In all plots, the light blue lines mark the $95 \%$ bootstrap confidence interval. The light blue column in all figures (300 s and $380 \mathrm{~s}$ ) marks the light stimulation period. 

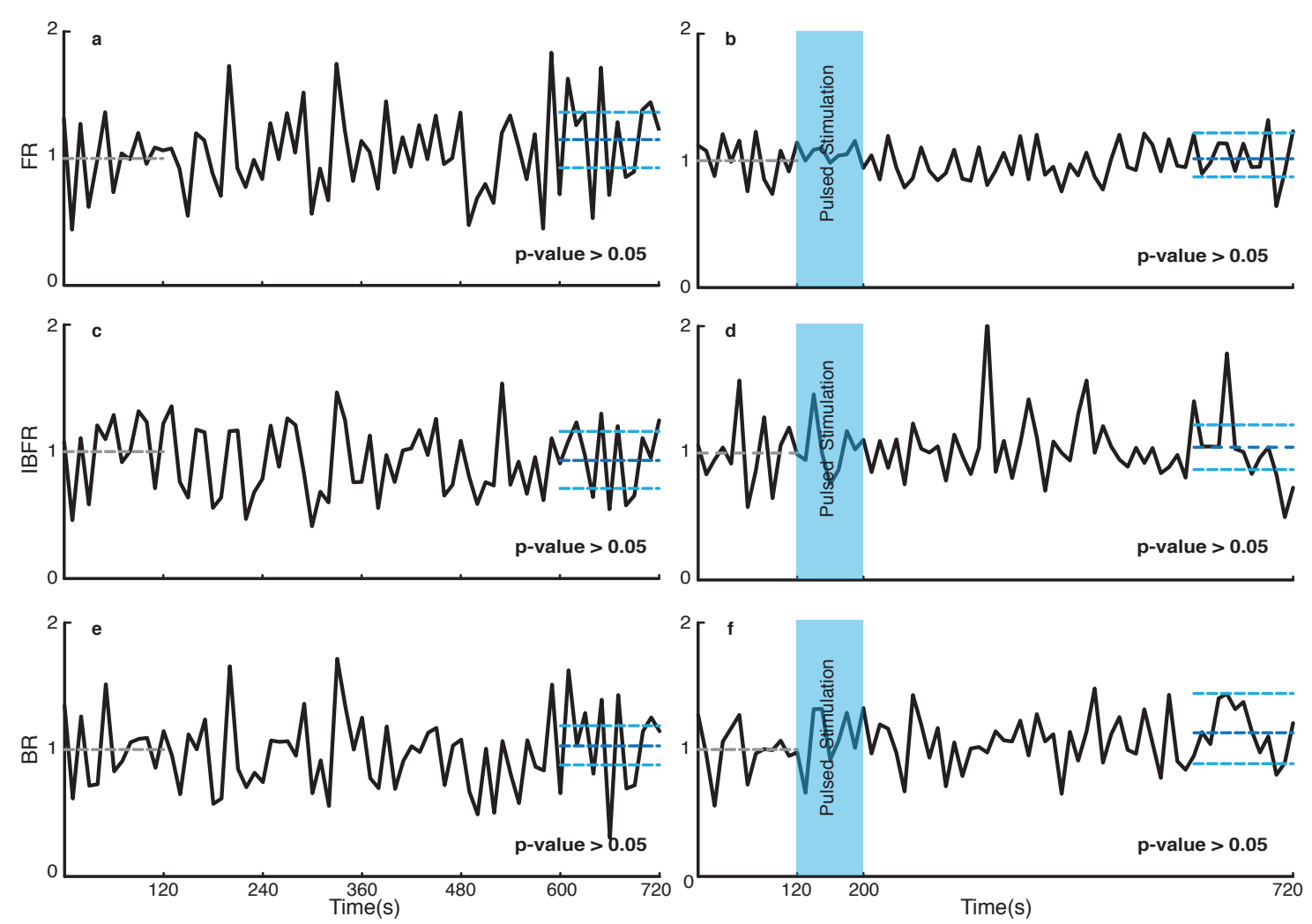

Figure 2.4: Control experiments. Plots on the left side are corresponding to control experiments of transduced cultures without light stimulation and the plots on the right side are for control experiments on non-transduced cultures stimulated with light. $(\mathrm{a}, \mathrm{b})$ Average normalized firing rate. Here and in the other panels, the dotted grey line marks the mean value of the first 2 minutes of recording which the activity of the culture is normalized to and the dark blue line marks the mean during the last 2 minutes of recording. (c,d) Average normalized intra-burst firing rate. (e,f) Average normalized burst rate. In all plots, the light blue lines mark the $95 \%$ bootstrap confidence interval. The light blue column in panels (b,d,f) (120 and $200 \mathrm{~s}$ ) marks the light stimulation period with pulsed stimulation protocol. P values on each plot give the significance level for the increase of firing rate, burst occurrence rate or intra-burst firing rate during the last 2 minutes of recording respectively. Results for the control experiments of transduced cultures without light stimulation are averaged over 7 experiments in 7 cultures. Results with stimulated non-transduced cultures are averaged over 5 experiments in 5 cultures. 


\subsubsection{Network bursting dynamics changes after stimu- lation}

Bursts are a characteristic of mature hippocampal cultures (Leinekugel et al., 2002). To specifically examine the properties of such bursts, we assessed the burst occurrence rate and the intra-burst firing rate, which describes the emerging network, burst structure. With both pulsed and fade-in stimulation, the average normalized burst occurrence rate and average normalized intra-burst firing rate substantially increased due to stimulation. In the case of pulsed stimulation, the average normalized intra-burst firing rate $(\mathrm{n}=18$ experiments) increased by $25 \%$ after stimulation compared to before stimulation (Fig. 2.3c) $\left(p<10^{-6}\right.$, Wilcoxon rank sum test). The average normalized burst occurrence rate $(\mathrm{n}=18$ experiments) increases by $21 \%$ after stimulation compared to before stimulation (Fig. 2.3p) $\left(p<10^{-5}\right.$, Wilcoxon's rank sum test). In case of fade-in stimulation, the average normalized intra-burst firing rate $(\mathrm{n}=16$ experiments) after stimulation had $38 \%$ increase compared to before stimulation (Fig. 2.3d) $\left(p<10^{-5}\right.$, Wilcoxon rank sum test). On the other hand, the average normalized burst occurrence rate $(\mathrm{n}=16$ experiments) increased $20 \%$ after stimulation compared to before stimulation (Fig. $2.3 \mathrm{f})\left(p<10^{-2}\right.$, Wilcoxon rank sum test). We conclude that mild whole field blue light stimulation can modify network bursting dynamics and that fadein stimulation with ramps of light had an effect at least as pronounced as pulsed stimulation. In Fig. $2.3 \mathrm{~s}, \mathrm{~h}$, an example of spontaneous activity before and after stimulus is shown, which depict a significant increase of firing rate, burst rate and intra-burst firing rate. No significant changes in the average normalized intra-burst firing rate and average normalized burst occurrence rate was found under control conditions (Fig. 2.4c,d,e,f).

To examine next whether photo-stimulation affected the process responsible for the termination of bursts we examined the burst duration distribution. Burst durations minimally changes after stimulation for both stimulation types (Fig. 2.5p,d). In case of pulsed stimulation, the mean burst duration before stimulation was $810 \pm 90 \mathrm{~ms}$ ( $\mathrm{n}=1084$ bursts), the mean burst duration after stimulation at the last 5 minutes of recording was $840 \pm 130 \mathrm{~ms}$ 
( $\mathrm{n}=1209$ bursts). In case of fade-in stimulation, the mean burst duration before stimulation was $1040 \pm 160 \mathrm{~ms}(\mathrm{n}=859$ bursts $)$ and the mean burst duration after stimulation at the last 5 minutes of recording was $970 \pm 145$ ms $(\mathrm{n}=939$ bursts $)$.
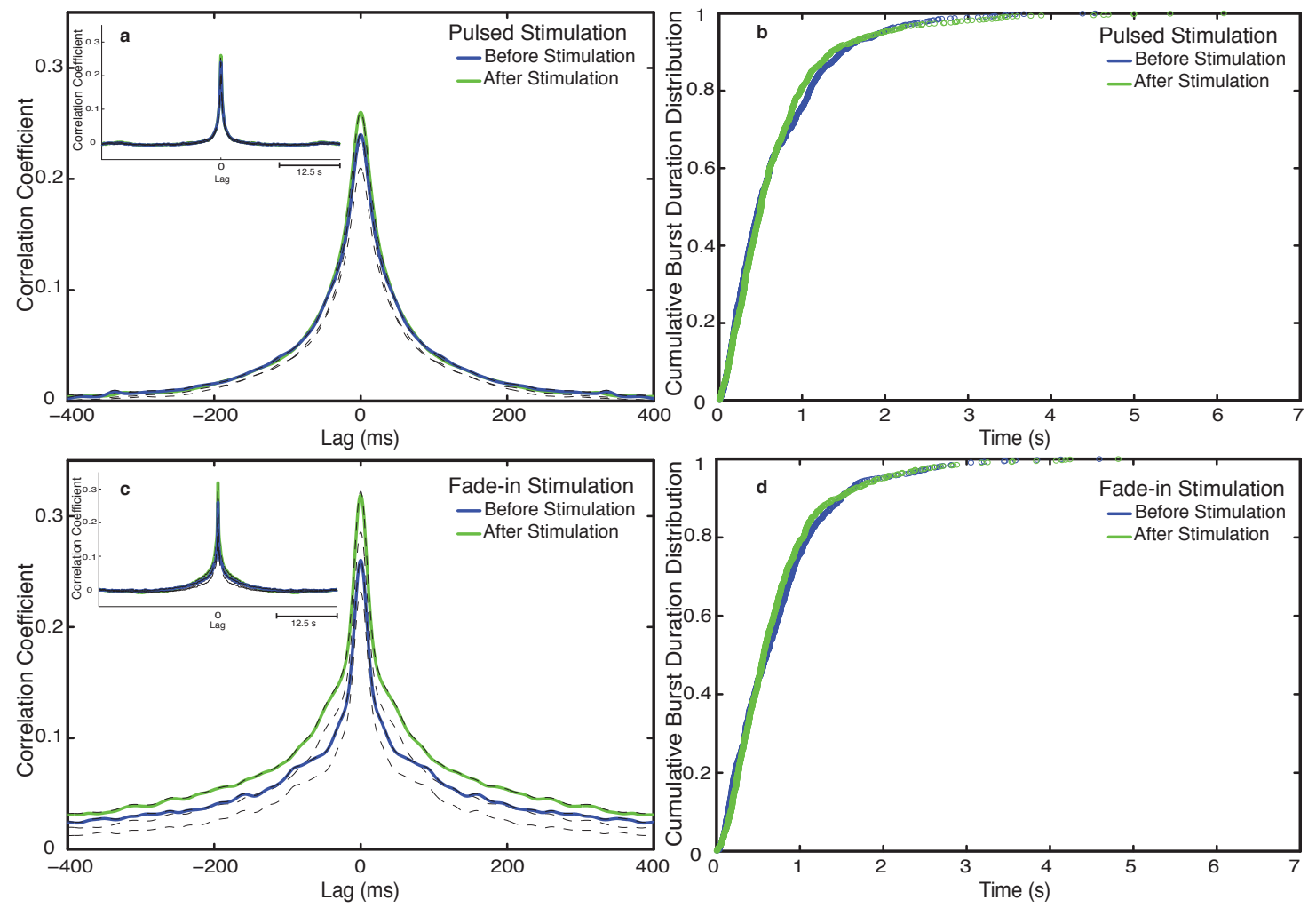

Figure 2.5: Changes in network synchronization. Panel (a) presents the average cross-correlation function of pulsed stimulation, with the blue line representing the average cross-correlation function before stimulation and the green line representing the averagecross correlation function after stimulation. Dotted lines mark Jackknife confidence intervals. The inset represents the long-term dynamics of the average cross-correlation function. Panel (b) represents the cumulative distributions of burst durations before stimulation (blue line) and after stimulation (green line) of pulsed stimulation. Panel (c) represents the cross correlation functions of fade-in stimulation. The inset represents the average cross-correlation function on a $1 \mathrm{sec}$ timescale. Panel (d) is the cumulative distribution of the burst duration before stimulation (blue line) and after stimulation (green line) of fade-in stimulation. 


\subsubsection{Interneuronal spike correlations increase after stim- ulation}

Although clearly demonstrating a substantial enhancement of collective network bursting, none of the quantitative indicators considered so far is sensitive to the detailed coordination of spike trains among the different neurons within the culture. We thus used cross-correlation functions in order to characterize changes in interneuronal synchronization after stimulation. To this end we computed the cross-correlation functions between multiunit spike trains recorded at different electrodes before and after stimulation (Fig. 2.5 a,c). Mathematically the average inter-electrode cross-correlation function is identical to the average cross-correlation function of the single neurons contributing to the compound spike trains. The half width at half maximum of the cross-correlation function is as follows: for pulsed stimulation, before the stimulus it is $52 \mathrm{~ms}$, and after stimulus it is $52 \mathrm{~ms}$, as for the fade-in stimulation before the stimulus it is $36 \mathrm{~ms}$ and after the stimulus it is $40 \mathrm{~ms}$. The half width at half maximum of all mean cross-correlation functions was thus much smaller than the mean burst duration confirming that the correlation functions indeed quantify intra-burst coordination of spiking among neurons. In the case of pulsed stimulation, the maximum cross correlation coefficient (at $\mathrm{t}=0$ ) increased from 0.24 before stimulation to 0.26 after stimulation. In the case of fade-in stimulation, the maximum cross correlation coefficient increased from 0.26 before stimulation to 0.31 after stimulation. In the case of pulsed stimulation ( $\mathrm{n}=2550$ pairs of electrodes in 18 experiments), the increase in the amplitude of the average cross correlation function was not statistically significant ( $p>0.05$, permutation test). On the other hand, in the case of fade-in stimulation ( $\mathrm{n}=2450$ pairs of electrodes in 16 experiments), we found a significant increase in the amplitude of the average cross-correlation functions compared to before stimulation $(p<0.01$, permutation test). Intriguingly, the enhancement of the average instantaneous cross-correlation was more pronounced in the case of fade-in stimulation than in the case of pulsed stimulation, further highlighting the effectiveness of mild photo-stimulation. 


\subsubsection{Optogenetic modification of network dynamics is NMDA- and AMPA- receptor dependent}

Hippocampal neuronal cultures consist primarily of pyramidal excitatory neurons $(\sim 80 \%)$ and, to a lesser extent, inhibitory interneurons $(\sim 20 \%)$. To identify the cellular basis of the observed enhancement of synchrony, we therefore tested the involvement of excitatory and inhibitory interactions in the network-level changes. To isolate the contribution of NMDA-receptordependent excitatory transmission, we used NBQX to block the activity of AMPA type glutamate receptors and Picrotoxin to block GABA $_{\mathrm{A}}$ receptor mediated inhibitory transmission throughout the recording session. We found that in these experiments the average normalized firing rate $(n=10$ experiments) increased by virtually the same factor of $27 \%$ after stimulation ( $p<10^{-5}$, Wilcoxon rank sum test) as in the previous experiments. Moreover, the average normalized burst occurrence rate and the average normalized intra-burst firing rate increases significantly by $23 \%$ and $19 \%$ respectively after stimulation $\left(p<10^{-3}\right.$ and $p<10^{-3}$, Wilcoxon rank sum test) (Fig. 2.6). This indicates that NMDA dependent synaptic transmission is sufficient to provoke the optogenetically induced level of changes. In order to study the potential involvement of AMPA receptors, we used APV and Picrotoxin to block NMDA- and $\mathrm{GABA}_{\mathrm{A}}$-receptors mediated transmission (Fig. 2.7). We found that in such AMPA receptor dominated networks the normalized average firing rate $(\mathrm{n}=10$ experiments) increased significantly by $8 \%$ after stimulation $\left(p<10^{-2}\right.$, Wilcoxon rank sum test). Moreover, both the average normalized burst occurrence rate $(\mathrm{n}=10$ experiments) and the average normalized intra-burst firing rate significantly increased by $36 \%$ and $15 \%$ respectively after stimulation (burst rate: $p<10^{-8}$, intra-burst firing rate: $p<10^{-6}$, Wilcoxon rank sum test). These results indicate that the observed change in the network dynamics is both AMPA and NMDA dependent. 

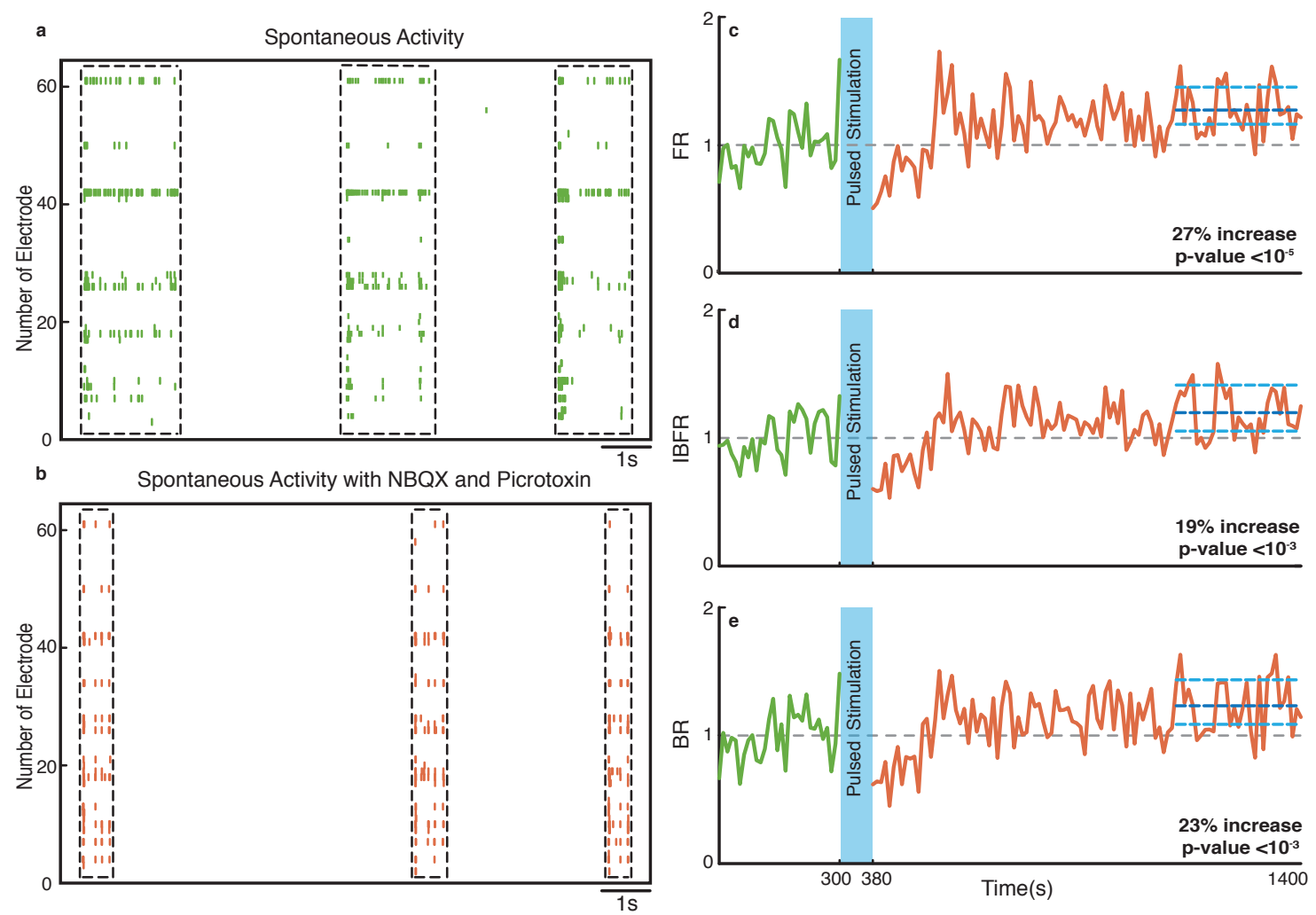

Figure 2.6: Network plasticity in the presence of NBQX/Picrotoxin. (a,b) Spontaneous activity (before stimulation) of a neuronal culture before and after adding NBQX/Picrotoxin. (c) Average normalized firing rate before and after stimulation. Here and in the other panels, the dotted grey line marks the mean before stimulation and the dark blue line marks the mean after stimulation (d) Normalized average intra-burst firing rate. (e) Normalized average burst rate. In all plots, the light blue lines mark the $95 \%$ bootstrap confidence interval. The light blue column in all figures (300 s and $380 \mathrm{~s}$ ) marks the light stimulation period. $\mathrm{P}$ values on each plot give the significance level for the increase of firing rate, burst occurrence rate or intra-burst firing rate during the last 5 minutes of recording respectively. The results are averaged over 10 experiments in 10 cultures. 

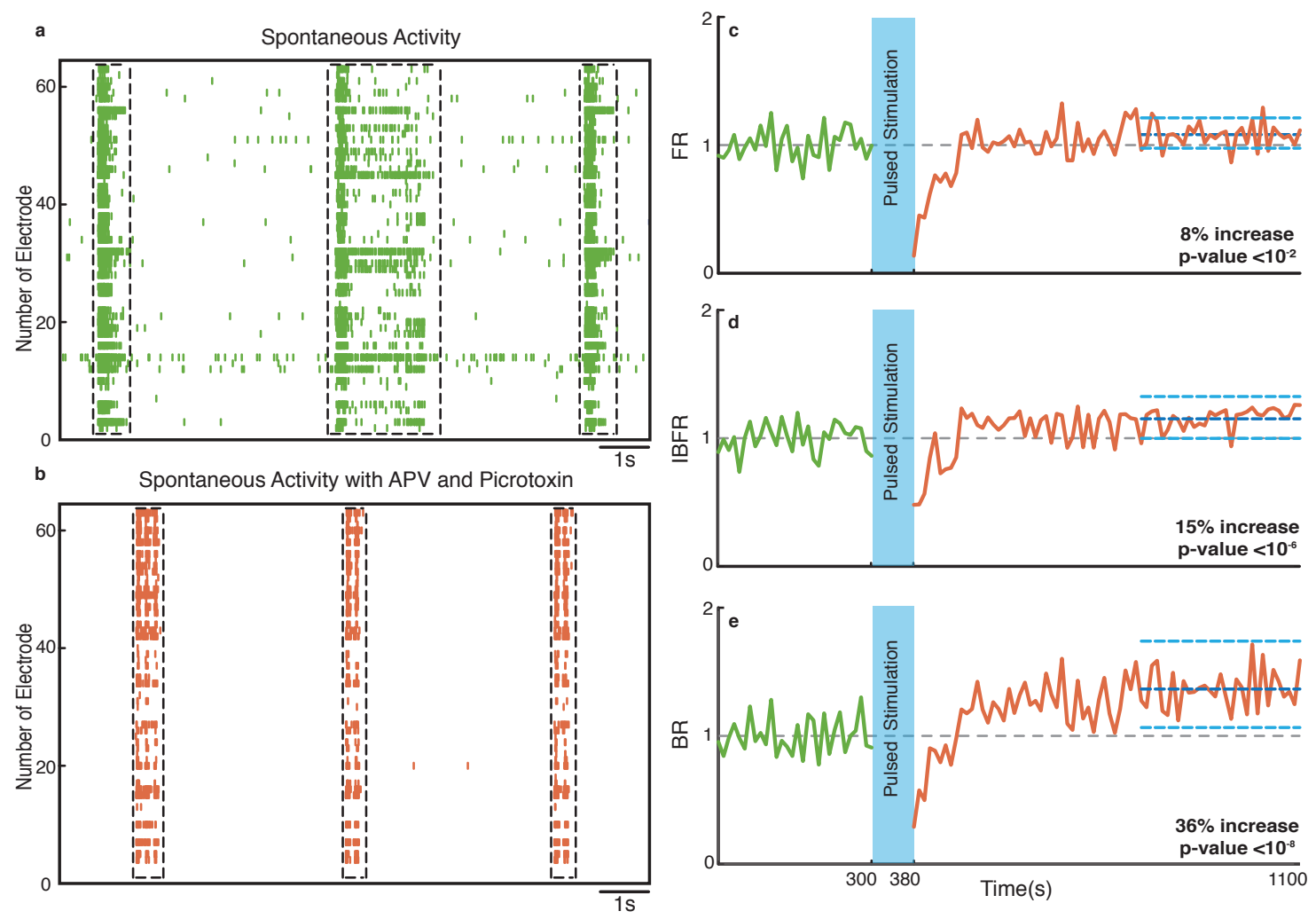

Figure 2.7: Network plasticity in the presence of APV/Picrotoxin. (a,b) Spontaneous activity (before stimulation) of a neuronal culture before and after adding APV/Picrotoxin. (c) Average normalized firing rate before and after stimulation Here and in the other panels, the dotted grey line marks the mean before stimulation and the dark blue line marks the mean after stimulation (d) Normalized average intra-burst firing rate. (e) Normalized average burst rate. In all plots, the light blue lines mark the $95 \%$ bootstrap confidence interval. The light blue column in all figures (300 s and $380 \mathrm{~s}$ ) marks the light stimulation period. $\mathrm{P}$ values on each plot give the significance level for the increase of firing rate, burst occurrence rate or intra-burst firing rate during the last 5 minutes of recording respectively. The results are averaged over 10 experiments in 10 cultures.

\subsection{Discussion}

Our results demonstrate that mild types of optogenetic stimulation, using low light power density and low frequency at which light pulses are delivered, are sufficient to induce global changes in neuronal network burst synchronization. Photo-stimulation of Channelrhodopsin-2 transducted hip- 
pocampal neuronal cultures increased firing rate, intra-burst firing rate, burst occurrence rate and interneuronal spike correlations. These changes in network dynamics appear to be mediated via a mixed mechanism involving both AMPA and NMDA receptors. Studying the duration and internal structure of 4091 synchronized network bursts in 34 cultures, we observed that the process terminating network bursts is virtually unaffected by optogenetic stimulation while the coordination among different neurons is selectively enhanced. Perhaps the most surprising result of our experiments was that the slowly increasing fade-in light stimulation, was in every respect at least as effective in reorganizing the network dynamics as the stronger pulsed stimulation protocol. It suggests that a small number of spiking events can more effectively induce changes of the collective dynamics than massive externally imposed activity patterns.

Overall, our results indicate that optical stimulation is a viable and powerful tool to examine network plasticity. Previously, studies of plasticity in neuronal cultures have primarily used electrical stimulation. Electrical stimulation has the disadvantage of producing substantial artifacts for MEA recordings (Wagenaar and Potter, 2002) and micro-electrodes are fixed in position "substrate embedded" so the stimulation sites are fixed. Thus, it is only possible to stimulate a small subset of neurons. In order to activate neuronal networks globally, alternative methods are needed. The only approaches previously available were the chemical induction methods that can activate many synapses simultaneously. Chemical induction, however, requires chronic treatment with pharmacological agents that might interfere with the physiological state of the neurons and offers no temporal control (Molnár, 2011). Alternative to chemical induction, Channelrhodopsin-2 has been used to induce plasticity at single synapses using $200 \mathrm{~ms}$ blue light pulses of frequency $0.5 \mathrm{~Hz}$ (Zhang et al., 2008). As a result of stimulation, a lasting increase of spine volume was showed accompanied by increased in $\alpha$ CamKII concentration. The aforementioned study has looked at the changes in the single neuron dynamics induced by an optogenetic plasticity induction protocol. Previous study by Dranias et al. (2013) has used random dot blue light stimuli in order to investigate short term plastic changes 
(short term memory) that were maintained for as long as 1 s in cultured Channelrhodopsin-2 transfected neuronal networks on MEAs. On the other hand, the study by Takahashi et al. (2012) has shown that repeated rhythmic low frequency photo-stimulation is more efficient to control the global activity of Channelrhodopsin-2. Our study complements the aforementioned studies combining optogenetics and multi-electrode array recordings, by looking into the long-term changes in bursting dynamics and interneuronal synchronization. We examined the network-level changes to a protocol of $0.5 \mathrm{~Hz}$ frequency, which has been previously shown to avoid network fatigue (Darbon et al., 2002).

The set of firing statistics examined in our analyses was sufficient to reveal the overall character of network reorganization. The network collective dynamics consistently changed after stimulation with respect to all three mean firing statistics: firing rate, intra-burst firing rate, and burst occurrence rate. Both the mean firing rate and the intra-burst firing rate increased substantially after offset of stimulation compared to the spontaneous activity of the culture as a result of network-level potentiation. The intra-burst firing rate increases with the same magnitude as the firing rate. As the majority of the spikes occur within the bursts, the increased firing rate is largely responsible for increased intra-burst firing rate. All of these features indicate a specific increase of network excitability due to enhanced excitatory interactions and a virtually unaffected mechanism of burst termination. Moreover, we found no significant changes in the average normalized intra-burst firing rate before and after stimulation in control experiments. This further confirms that the change in the synchronized activity of the culture is due to the plastic changes in synaptic interactions, rather than due to membrane potential fluctuations. Our results are consistent with findings from previous studies that used electrical stimulation. Maeda et al. (1998) for instance were able to induce an increase of the burst occurrence rate and the intra-burst firing rate using high frequency tetanic stimulation. In comparison, our stimulation protocol is able to induce lasting potentiation without a need for high frequency stimulation, which might exhaust the network. Some of the changes reported previously on bursting dynamics using electrical stimulation appeared more pronounced 
than our findings. This might reflect differences in induction protocol or the relatively small data sets. The large size of the data set analyzed here makes it quite easy to identify and characterize changes in network dynamics with good sensitivity and precision.

Our pharmacological analyses indicate that the network-level potentiation described here is mediated via a combined NMDA/AMPA receptor mechanism. It is important to note that we used a combination of blockers (APV/Picrotoxin or NBQX/Picrotoxin), as the addition of APV or NBQX alone to the MEA chamber completely abolishes the synchronized bursting activity while the use of Picrotoxin tremendously increased the activity making it hard to observe any changes in the collective network dynamics. Although we only investigated the possible plasticity mechanisms that underlie the observed changes in network dynamics, this does not exclude the possibility of the involvement of cellular excitability changes that needs to be tackled in a follow up study. It is important to mention that none of the pharmacological blockers we used when applied acutely have effects on cellular excitability. On the other hand, chronic exposure to blockers might lead to changes in cellular excitability e.g. synaptic NMDA receptors blockade beyond three hours have effects on intrinsic excitability changes (personal communication, Oliver Schlüter). Nevertheless, it is important to note that plasticity changes and cellular excitability changes are tightly intertwined as they share common induction mechanisms. EPSP-spike potentiation requires the activation of NMDAR for its induction (Jester et al., 1995; Daoudal et al., 2002; Wang et al., 2003) sharing a common induction pathway with LTP. NMDAR is not the only glutamate receptor that participates in the induction of long lasting excitability plasticity. mGluR is also involved in the induction of long-term synaptic plasticity in the hippocampus and also involved in the induction of long lasting intrinsic excitability plasticity. Studies have confirmed that there are common features linking the synaptic plasticity and intrinsic plasticity. EPSP-spike plasticity in the CA1 area of the hippocampus is particularly good example of EPSP-spike potentiation and is observed when LTP is induced homosynaptically (Bliss and Lømo, 1973; Abraham et al., 1987; Daoudal et al., 2002). If the activation of a synaptic 
receptor was not directly involved in the induction of plasticity, postsynaptic depolarization was a determining factor, and calcium elevation was necessary (Aizenman and Linden, 2000; Tsubokawa et al., 2000). Downstream of calcium elevation, several protein kinases and phosphatases (e.g. CaMKII, PKC, PKA) that play a central role in synaptic plasticity (Lisman, 1994) are also involved in the induction of several activity dependent forms of intrinsic plasticity (Ganguly et al., 2000; Tsubokawa et al., 2000; Wang et al., 2003). These kinases and phosphatases are also known to have various activities on $\mathrm{Na}^{+}$channels, $\mathrm{Ca}^{+}$channels, $\mathrm{K}^{+}$channels and cationic $\mathrm{I}_{\mathrm{h}}$ channels (Cathala and Paupardin-Tritsch, 1997; Herzig and Neumann, 2000; Cantrell and Catterall, 2001; Schrader et al., 2002). In addition, these factors may also regulate targeting and recycling of many ion channels at the plasma membrane (Dargent et al., 1995; Tanemoto et al., 2002; Hu et al., 2003). These aforementioned complex mechanisms require an extensive follow up study to delineate the contribution of synaptic and/or intrinsic excitability plasticity in the context of the photo-stimulation induced network-level potentiation that we observed.

In our experiments, we also examined changes in correlation structure of the network after offset of stimulation. We found an increase in the amplitude of cross-correlation functions after stimulation reflecting an increase in spike synchronization. Significant cross-correlations can arise in the presence of direct synaptic connections and/or from common or correlated inputs between pairs of neurons (Ostojic et al., 2009). The amplitudes of the crosscorrelations not only depend on the properties of the synapses involved, but are also modulated by the general activity of the neurons (Ostojic et al., 2009; Tchumatchenko et al., 2010; Battaglia et al., 2012). Precise spike timing is known to be essential for many forms of synaptic plasticity (Caporale and Dan, 2008). The increase in spike synchronization that we observed is likely to reflect tighter coupling between neurons rather than a change in the overall activity level of burst firing. The width of the cross-correlation functions was generally much smaller than the mean burst duration either before or after stimulation for both pulsed and fade-in photo-stimulation. This demonstrates that the change in correlation structure results from modifications in 
the fine structure within the burst. The half width at half maximum of the cross-correlation function is on the order of $50 \mathrm{~ms}$, which is on the order of the decay time constant of NMDA receptor mediated synaptic currents constant. The aforementioned suggests that the enhancement of correlations under all conditions can be explained by an enhancement of common input that has a substantial NMDA receptor component. Our results are consistent with the increased spike correlations that have been observed in the case of hippocampal neurons subjected to a chemical plasticity induction method (Ivenshitz and Segal, 2006). Our correlation results highlight again the sensitivity gained by harnessing the potential of high yield network electrophysiology, combining optogenetic stimulation and multi-electrode recordings, that allows efficient gathering of large data sets for a precise, stable and reliable characterization of network dynamics.

In conclusion, we presented a simple and effective photo-stimulation protocol able to modify the intrinsic collective dynamics of collective network bursts, substantially enhancing spike synchronization. It provides a qualitative alternative to stimulation protocols that externally enforce modified activity patterns onto neuronal networks. Modifying network synchronization can be expected to be relevant in studying activity dependent developmental processes, where the correlation structure of neural activity, as in the visual pathway (Weliky, 1999), is crucial. For such application, modifying the intrinsic ability of a network to generate correlated activity patterns might often be preferable to permanently impose desired activity patterns from the outside. We are confident that the approach presented here will substantially aid in the search for a photo-stimulation protocols that strengthen, reduce or abolish network synchronization, building a toolbox for modifying collective neuronal network dynamics. 


\section{Chapter 3}

\section{Enhancing burst activation and propagation in cultured neuronal networks by photo-stimulation}

\subsection{Introduction}

Synchronized burst activity is an emergent property of the cultured neuronal networks in-vitro (Maeda et al., 1995; Wagenaar et al., 2006a). This synchronized activity appears almost as early as the network is connected physically (about 2 days in-vitro). The synchronization level increase with higher age of the culture, until at about a week in-vitro, when the network finally displays spiking activity that recruits a large fraction of neurons in the network for a few hundred milliseconds of high frequency, synchronous firing (Maeda et al., 1995; Cohen et al., 2008). It has been shown that the order of activation within a synchronized burst is hierarchical rather than random (Eytan and Marom, 2006). It was shown that a small subset of neurons, called leader neurons, are initiating a majority of network bursts (Ham et al., 2008) and they are consistently firing tens of milliseconds before others (Eytan and Marom 2006). Such leader neurons create network bursts by recruiting follower neurons. 
As shown in Chapter 2, the intrinsically generated network synchrony can be enhanced effectively by using optogenetics. In this Chapter, the indication of the aforementioned changes in the initiation and propagation of the network synchronized activity is investigated via the leader-follower relationship.

\subsubsection{Leaders and Followers}

In order to study the ignition of collective spontaneous activity, the easiest is to start with one-dimensional (1-D) cultures, by restricting neurons to grow along 1-D patterns lines (Feinerman et al. $2005 \& 2007$ ). In this case, the firing pattern of such simplified systems can be well understood in terms of a generation process that occurs in one of several burst initiation zones (BIZ), and a subsequent propagation from that area into the rest of the line. The major advantage of using 1-D cultures to study ignition and propagation is that a signal has only a limited pathway.

Two-dimensional (2-D) networks exhibit similar activity, but may have a large number of possible paths connecting any two given points, which makes the situation much less clear (Eckmann et al., 2008). Empirical evidences invitro (van Pelt et al., 2004; van Pelt et al., 2005) as well as in-vivo (Tsodyks et al., 1999) indicated that the sequence of neuronal activation within a synchronization event is nonrandom and strongly constrained by the pattern of population activity. Previous theoretical and experimental research showed that in 2-D cultures there are also a select group of neurons, termed initiation loci (Maeda et al., 1995), privileged or leader neurons (Eytan and Marom, 2006; Eckmann et al., 2008), or major burst leader (MBL) (Ham et al., 2008), ignite and create network bursts by recruiting constituent neurons termed as followers (Ham et al., 2008).

It is shown that the leader neurons, even in variety of cultures, can remain stable over long times when the culture is mature and spontaneously active (Ham et al., 2008; Eckmann et al., 2008). Moreover, Eckmann et al. (2008), also tried to alter the leader distribution by using electrical stimulation, but the distribution has stabilized within about $1 \mathrm{~h}$. Despite showing the exis- 
tence and robustness of leader neurons as ignition sites to generate and propagate a synchronized network burst activity, many questions regarding the effect of stimulation on leader-follower relationships are still not settled. The first general question is whether stimulation can modify the leader-follower relationships.

In 1949, in a very influential postulate on the cellular basis for learning, Hebb stated that "when an axon of cell A is near enough to excite a cell B and repeatedly or persistently takes part in firing it, some growth process or metabolic change takes place in one or both cells such that A's efficiency, as one of the cells firing B, is increased". This formulation suggests a potential causal relation between the firing of the two neurons, which requires that the presynaptic neuron fires slightly before the postsynaptic one. This postulate gained strong experimental support with the finding of long-term potentiation (LTP) of synaptic transmission, initially discovered in the hippocampus (Bliss and Gardner-Medwin, 1973; Bliss and Lømo, 1973). One of the first experimental investigations to study this temporal requirement for the coincidence of pre- and postsynaptic activity to induce synaptic potentiation is the study of Levy and Steward in 1983. Subsequent studies further demonstrated the importance of the temporal order of pre- and postsynaptic spiking in synaptic modification (Bi and Poo, 1998; Markram et al., 1997; Debanne et al., 1998; Zhang et al., 1998), known as spike-timing dependent plasticity (STDP). Thus, STDP captures the importance of causality in determining the direction of synaptic modification, which is implied in Hebb's original postulate. This causality requires that the presynaptic neuron fires slightly before (in case of LTP) or after (in case of LTD) postsynaptic one. Typically this plasticity requires multiple pre-post spike pairs. As mentioned before, we want to investigate whether stimulation can modify the leader-follower relationship. More precise question in this respect is whether it is necessarily required to use specific spatiotemporal paired stimulation like STDP or it is also possible by using unspecific global stimulation. To address this question, the global whole field pulsed and fade-in stimulation paradigms are used in this study. 


\subsubsection{Aim of the study}

In this study, we investigated the leader-follower neurons dynamics modifications using a combination of multi-electrode array and Channelrhodopsin-2 transduced hippocampal neuronal cultures. Using fade-in and pulsed photostimulation paradigms, we established that network-level potentiation is possible even by using unspecific global stimulation. The leader-follower dynamics are differentially modulated by different photo-stimulation paradigms. It is shown that fade-in stimulation can substantially affect leader-follower dynamics rather than pulsed stimulation. The network-level potentiation after fade-in stimulation is particularly reflected in a tighter leader-follower neurons relationship. Our results indicate that slowly fading-in light stimulation can be even more effective in potentiating network-level plasticity.

\subsection{Results}

Our experimental setup, as described in detail in Chapter 4, combines multichannel recording using multi-electrode arrays and whole field photo-stimulation. Fig. 3.1p shows a 21 DIV embryonic hippocampal neurons plated on 60 channels multi-electrode array (MEA) transduced with an AAV1/2-ChR2-YFP virus (Petreanu et al. 2009, Suska et al 2013).

Here, in order to study the effect of stimulation on leader and follower neurons, only the bursts initiated via one of the leader electrodes are taken into account for the whole analysis. Leader electrodes are mainly robust even after photo-stimulation.

\subsubsection{Changes in the IBFR maximum peak after photo- stimulation}

A typical recording obtained from one culture and the used photo-stimulation protocols (1) fade-in and (2) pulsed are presented in Fig. 3.19 and Fig. 3.2 a. A raster plot of one sample burst before and after fade-in stimulation of 
one experiment is shown in Fig. 3.1k, d, which shows how follower electrodes start to fire earlier, after initiation of the burst via leader electrode, after offset of stimulation compared to before. Similarly, in Fig. 3.2 , d, a raster plot of one sample burst before and after pulsed stimulation is shown, which shows no significant difference in the initiation of firing via follower electrodes, after initiation of the burst via leader electrode, after offset of stimulation compared to before. The depicted electrode spike trains here and all our other experiments typically represent multiunit activity as no attempt for spike sorting was made. Moreover, as previously shown, during the stimulation, the network responded as expected to the blue light stimulation in different manner to fade-in and pulsed stimulation (Fig. $3.2 \mathrm{~b}$ ).

We investigated the intricate details of the network level enhanced activity by comparing the maximum peak of the IBFR at each electrode before and after stimulus. Our analysis was done for both leader and follower electrodes. In case of fade-in stimulation (Fig. 3.3a,b), the maximum peak of IBFR after stimulation for 125 follower and 35 leader electrodes increased significantly compared to unperturbed spontaneous activity (followers and leaders respectively: $p=0.01, p<10^{-2}$, Wilcoxon signed rank test). As for pulsed stimulation (Fig. 3.3.,d), the maximum peak of IBFR after stimulation for 132 follower and 29 leader electrodes also increased substantially compared to unperturbed spontaneous activity prior to stimulation (followers and leaders respectively: $p<10^{-5}, p=0.02$, Wilcoxon signed rank test). Moreover, cumulative distribution of the ratio of the maximum peak of IBFR after to before stimulation showed a significant difference between fade-in and pulsed stimulation (Fig. 3.5a) ( $p=0.02$, Wilcoxon rank sum test). 


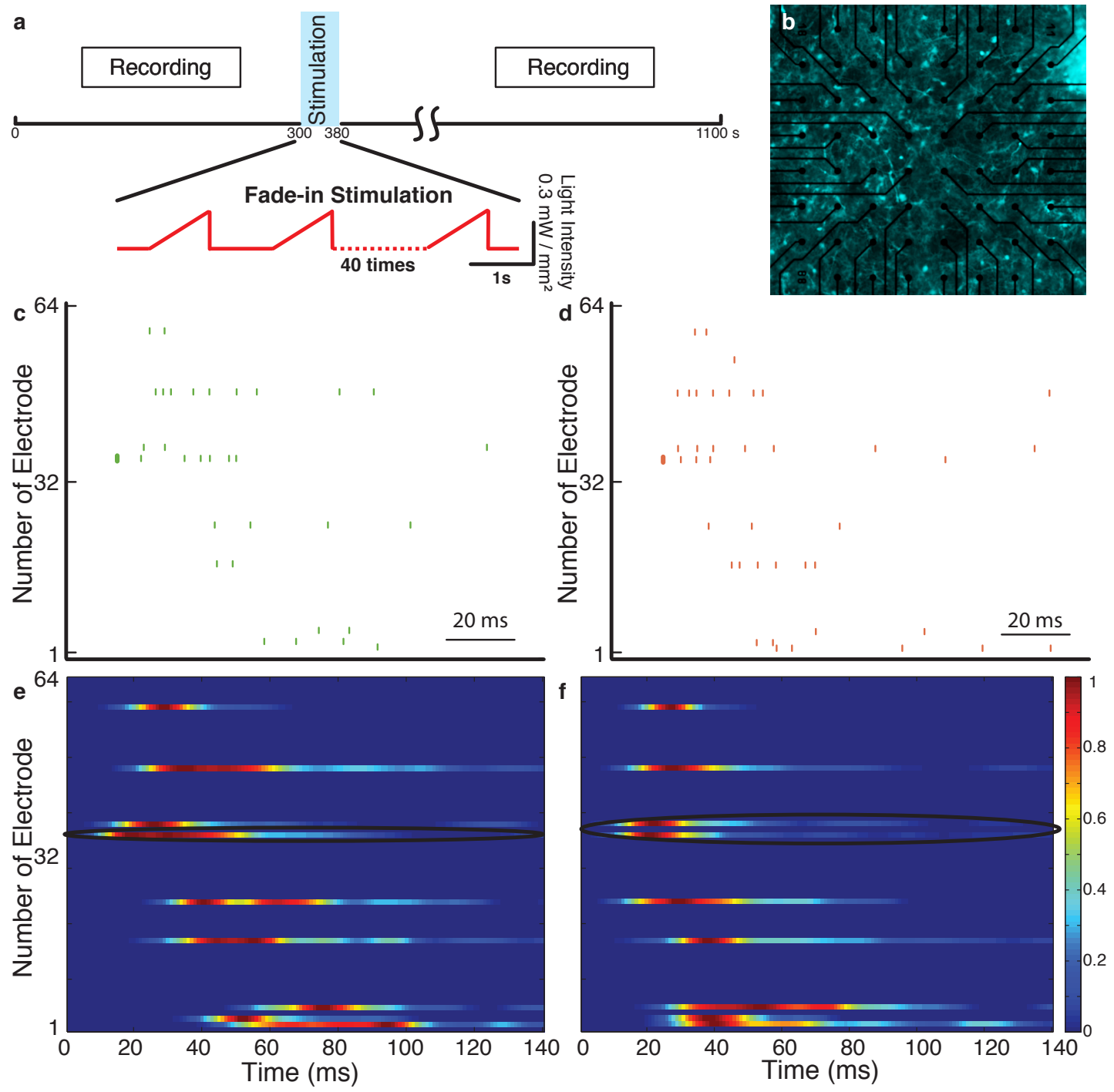

Figure 3.1: Fade-in stimulation. Panel (a) depicts the recording and photostimulation design with fade-in stimulation. Panel (b) shows the Channelrhodopsin-2 transduced neurons cultured on multi-electrode array. In panels $(\mathrm{c}, \mathrm{d})$ an example of one burst before and after fade-in stimulation which are initiated via the leader electrode is shown. The first spike fired from the leader electrode is bolded. The average normalized IBFR over all bursts of the corresponding sample example are shown in panels (e,f). The leader electrode is shown in these panels with black ellipse. There was 9 active electrodes in this example. The silent electrodes are shown in dark blue which shows zero-level of activity. 

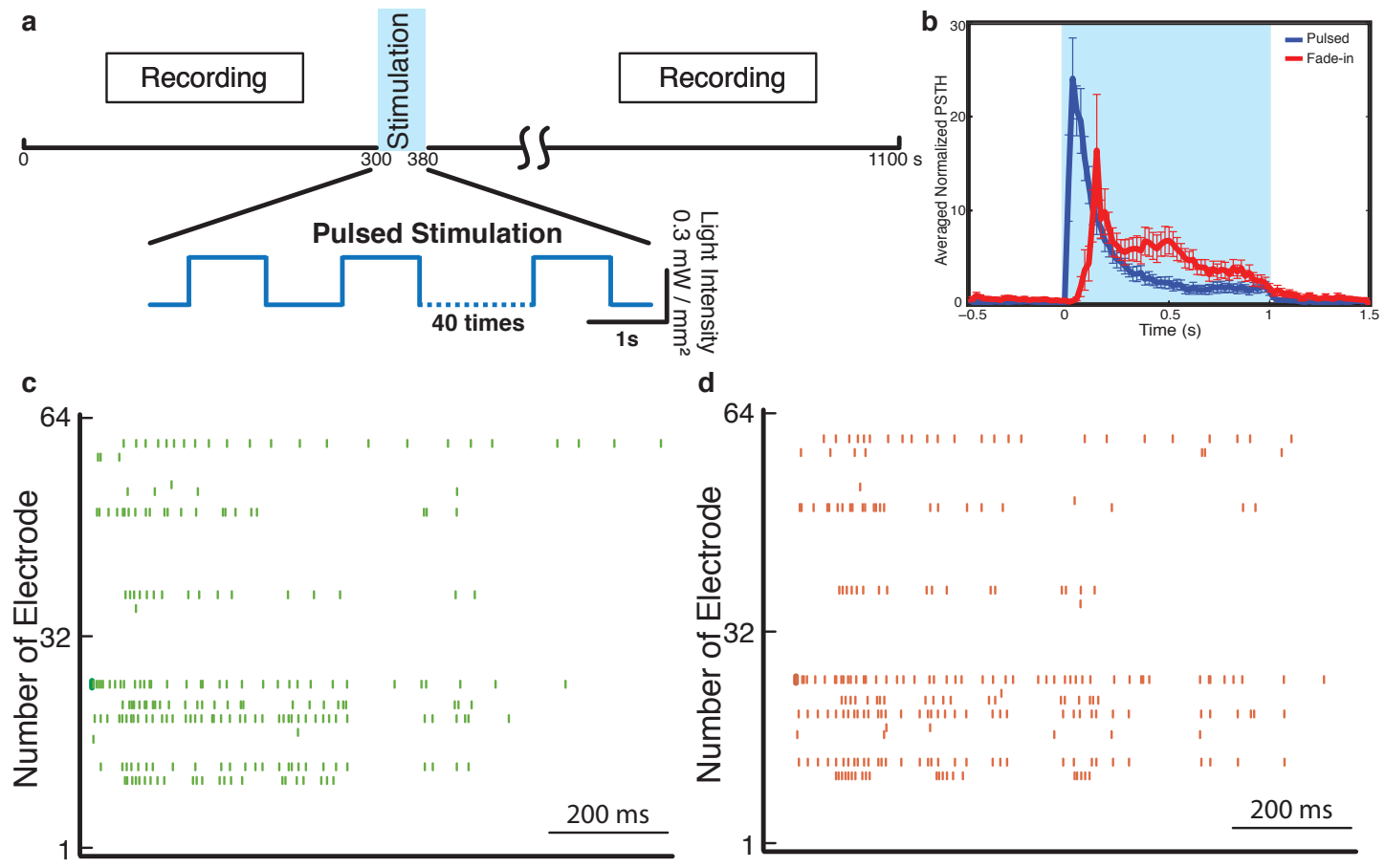

Figure 3.2: Pulsed stimulation. Panel (a) depicts the recording and photostimulation design with pulsed stimulation. Panel (b) presents the electrode averaged normalized peri-stimulus time histogram (PSTH) for both pulsed (dark blue) and fade-in stimulation (red). In panels (c,d) an example of one burst before and after pulsed stimulation which are initiated via the leader electrode is shown. The first spike fired from the leader electrode is bolded. 

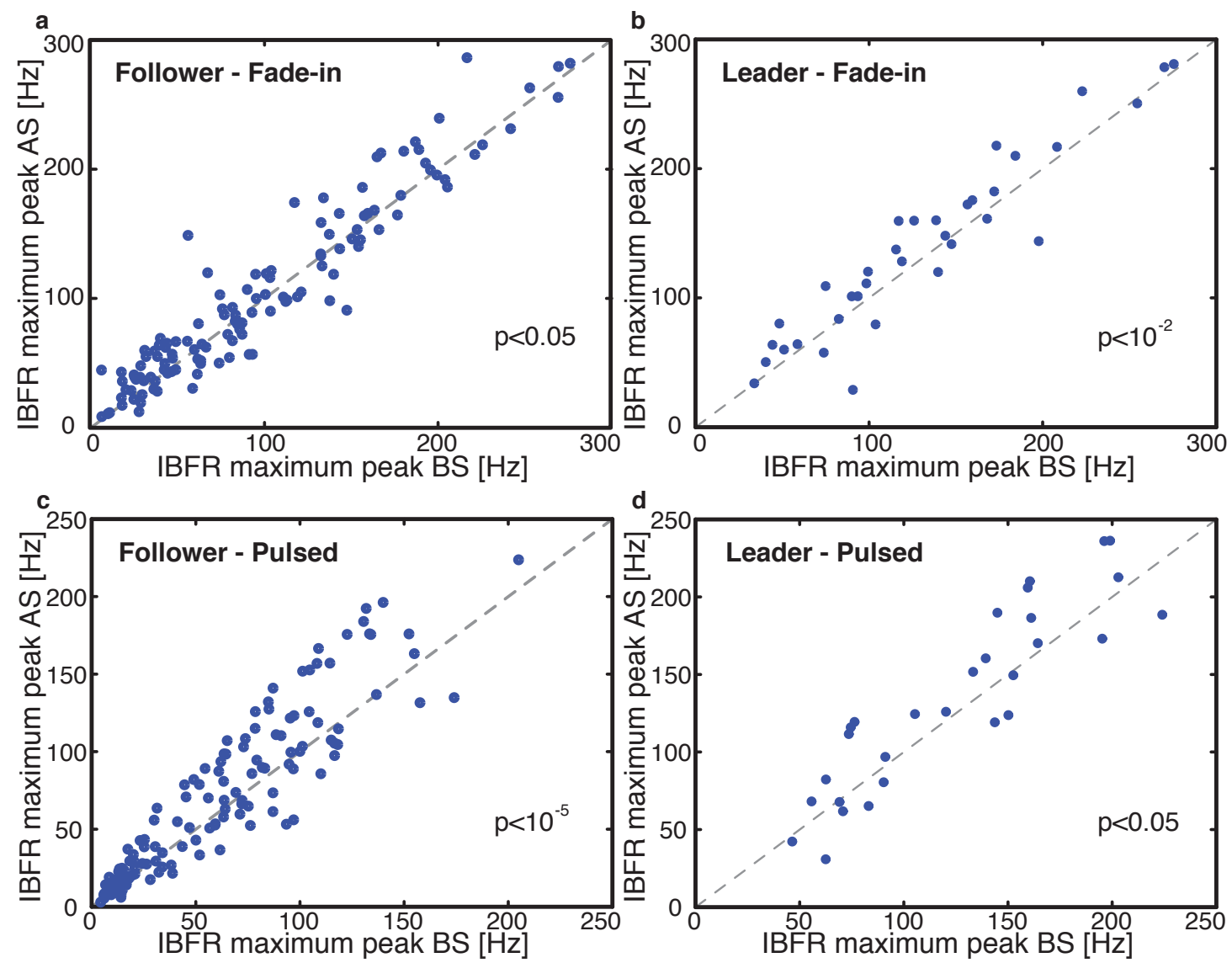

Figure 3.3: Intra-burst firing rate (IBFR) maximum peak. Panel (a,b) shows significant increase of IBFR maximum peak of follower and leader electrodes after fade-in stimulation compared to before. Panel $(\mathrm{c}, \mathrm{d})$ shows significant increase of IBFR maximum peak of follower and leader electrodes after pulsed stimulation compared to before. For pair comparisons the Wilcoxon signed rank test was used.

\subsubsection{Changes in the IBFR maximum peak delay after photo-stimulation}

We also have compared the time delay of the IBFR peaks from the onset of the burst. A raster plot of one sample burst before and after fade-in stimulation of one experiment is shown in Fig. 3.1p,d, which shows how follower electrodes start to fire earlier, after initiation of the burst via leader electrode, after offset of stimulation compared to before. The normalized IBFRs 
of AEs over all bursts initiated via the leader electrodes of the same experiment before and after fade-in stimulation shows a shorter peak delay after stimulation (Fig. 3.1 ,f). In Fig. 3.4a, the peak delay scatter plot of 125 follower electrodes from 12 experiments shows that the peak delay of follower electrodes gets significantly shorter after fade-in stimulation compared to the unperturbed spontaneous activity $\left(p<10^{-5}\right.$, Wilcoxon signed rank test). However, in case of leader electrodes (35 electrodes in total from 12 experiments) no significant change in peak delay after stimulation is observed (Fig. $3.4 \mathrm{~b})$.

In case of pulsed stimulation, no significant change in peak delay of follower and leader electrodes was observed after offset of the stimulation compared to unperturbed spontaneous activity (Fig. 3.4c,d) (132 follower electrodes and 29 leader electrodes from $\mathrm{n}=12$ experiments) $(p>0.05$ in both cases, Wilcoxon signed rank test).

In order to compare the change of peak delays after offset of the stimulation between pulsed and fade-in stimulation, the cumulative distribution of the difference between peak delay after and before stimulation is shown in Fig. 3.5b ( $p<10^{-3}$, Wilcoxon rank sum test). This shows that with fade-in stimulation the decrease in peak delays of IBFRs after stimulus is much more pronounced compared to pulsed stimulation. 

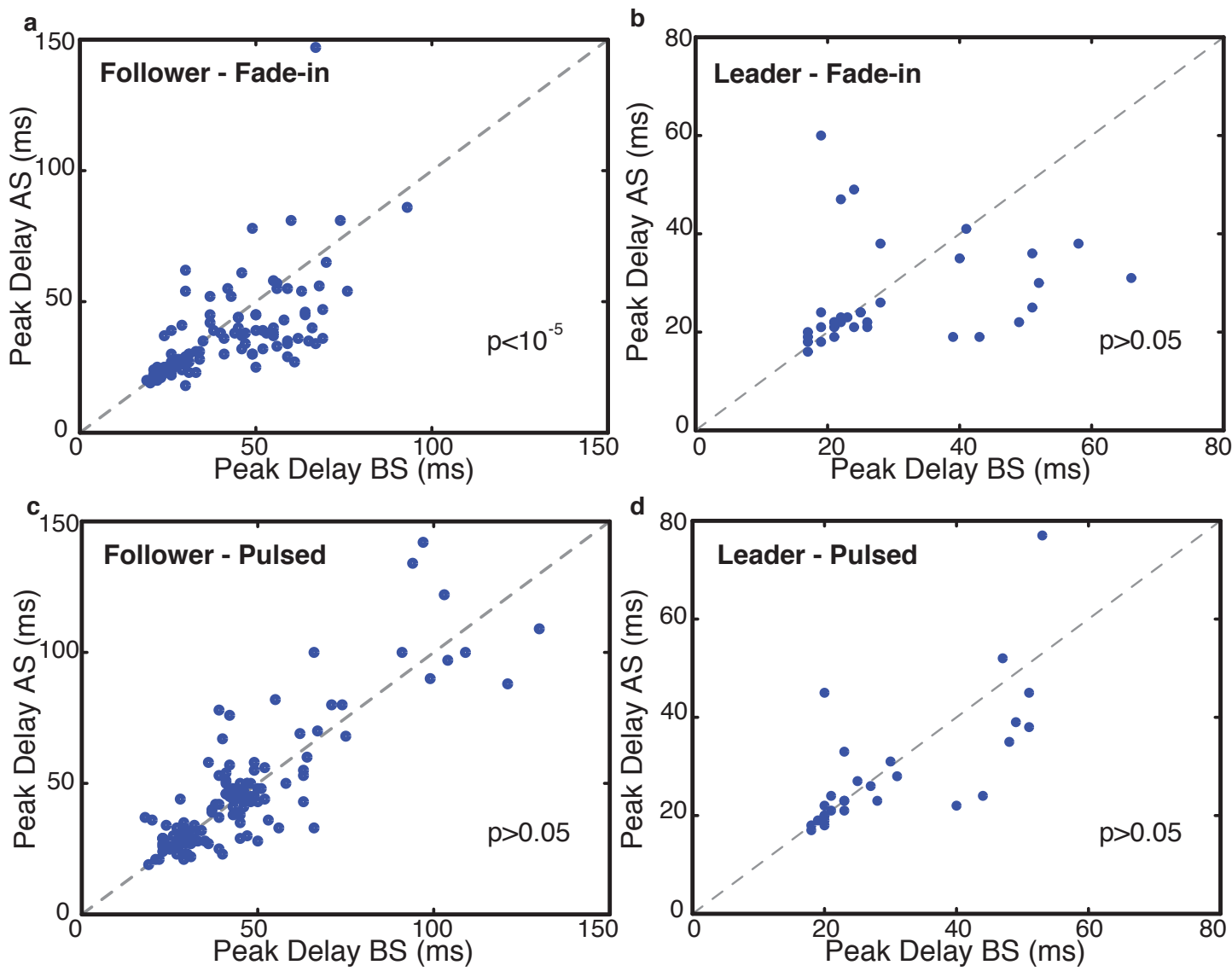

Figure 3.4: Peak delay. Panel (a,b) shows that in case of fade-in stimulation, the peak delay after stimulus decrease significantly compared to before stimulus $\left(\mathrm{p}<10^{-5}\right)$ and no significant difference is observed in case of leader electrodes $(\mathrm{p}>0.05)$. Panel $(\mathrm{c}, \mathrm{d})$ shows that the peak delay after stimulus has no significant change either for followers or leaders ( $\mathrm{p}>0.05)$. For pair comparisons the Wilcoxon signed rank test was used. 

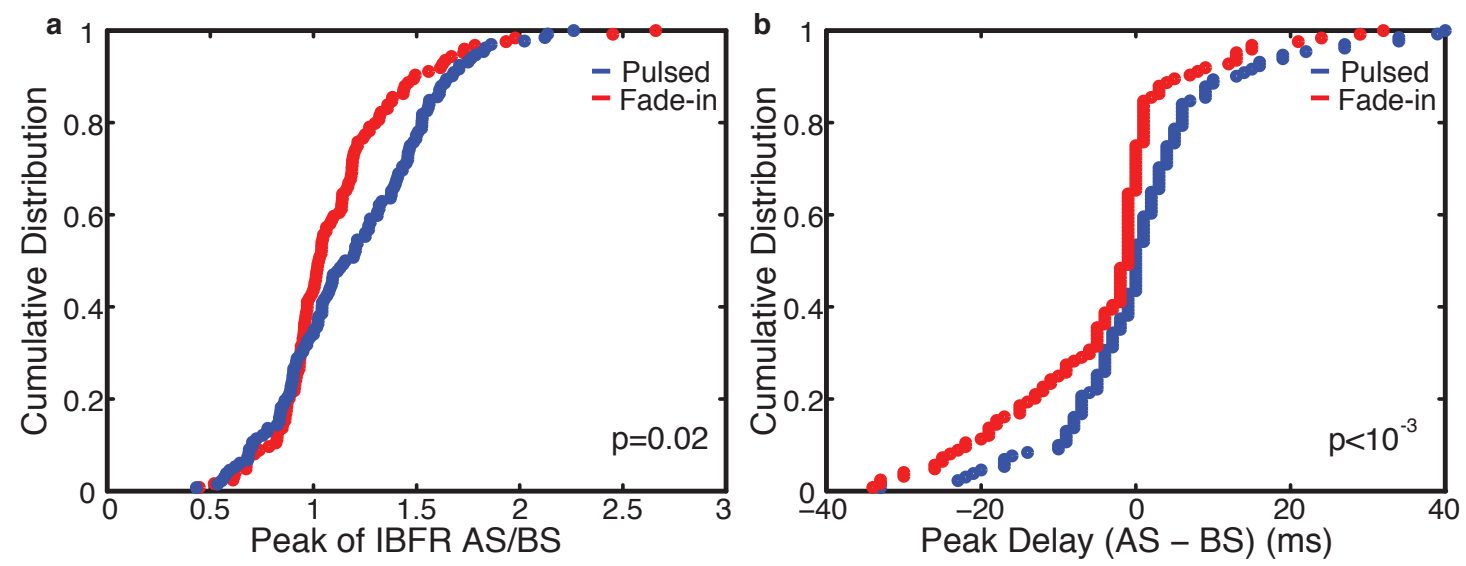

Figure 3.5: Comparisons between fade-in and pulsed stimulation. Panel (a) shows a significant difference between fade-in and pulsed stimulation cumulative distribution of the ratio of the maximum peak of IBFR after to before stimulation $(\mathrm{p}=0.02)$. Panel (b) shows a significant difference between fade-in and pulsed peak delay difference after and before $\left(\mathrm{p}<10^{-3}\right)$. For comparisons between cumulative distributions the Wilcoxon rank sum test is used.

\subsection{Discussion}

Using multi-electrode arrays, we recorded the synchronized activity of invitro cultured hippocampal networks. In combination with optogenetic stimulation using fade-in and pulsed photo-stimulation, we were able to modify the propagation of activity at burst onset across neurons. As the collective network dynamics is dominated by bursts, the intricate structure of the bursts should reflect the relationships between neurons and would be expected to change as a result of stimulation. In this study, we found that the leader-follower neurons relationship gets tighter after fade-in photostimulation.

The synchronized burst initiation involves two distinct processes: the activation of neurons and the recruitment of follower neurons by leader neurons into the burst. The activation process of the bursts has been reported to be a stereotypical process involving leader neurons that recruit follower neurons to participate in the burst and serves as a nucleation center for activity. In 
our recordings, leader neurons were mainly robust and did not change upon stimulation, which has been confirmed in other studies that pinpointed to the robustness of the first-to-fire neurons (Eckmann et al., 2008). The aforementioned suggest that the neuronal activation patterns follow a stereotypical hierarchical sequence and that the activation and recruitment within this sequence can be accelerated using photo-stimulation.

Eckmann et al. (2008) have shown that leader neurons remain stable over long times. They could alter the leader distribution, but the distribution was recovered within about 1 h. Our study presents further evidence that the leader neurons are mainly robust. The changes that were induced in the network are reflected in the relationship between leader and follower neurons and not the identity of leader and follower neurons per se. Leader neurons might be regarded as hub neurons as have been reported before to be involved in the initiation of population bursts (Czarnecki et al., 2012). Moreover, hub neurons have been reported to form a connected network that initiates the synchronized bursting (Quilichini et al., 2012) in the same manner in which leader neurons have been proposed to form a distinct sub-network among themselves (Eckmann et al., 2008).

We have shown that the maximum peak of intra-burst firing rate of leader and follower electrodes increases significantly after stimulation for both fade-in and pulsed stimulation. Interestingly, we found that the increase in the maximum of the intra-burst firing rate in case of the pulsed stimulation is stronger than in the case of fade-in stimulation. We have found that the normalized IBFRs of AEs over all bursts initiated via the leader electrodes of the same experiment before and after fade-in stimulation shows a shorter peak delay after stimulation reflecting that the follower neurons fire much faster in relation to the leader neurons, thus they get into a tighter relationship with the leader in the neuronal recruitment sequence. In case of pulsed stimulation, no significant change in peak delay of follower and leader electrodes is observed after offset of the stimulation compared to unperturbed spontaneous activity reflecting the fact that the temporal relationship between leader and follower neurons within the recruitment sequence is not tremendously affected. With 
fade-in stimulation the decrease in peak delays of IBFRs after stimulation is much more pronounced compared to pulsed stimulation indicating that fade-in photo-stimulation tightens the temporal relationship between leaders and followers in a much more pronounced manner. The tightening of the leader-follower relationship might be due to synaptic strengthening in a similar manner as in spike-timing dependent plasticity (Dan and Poo 2004) owing to the enhancement of inter-neuronal correlations during fade-in stimulation or due to the increased number of spikes within the bursts as a result of photo-stimulation.

Plastic changes in a network involve the modification of connections between neurons (Kruskal et al., 2013). The modification of those connections can be unraveled by looking at the effective connectivity between neurons (Rebesco et al., 2010) or by studying the changes undergone in the hierarchical neuronal activation pattern that initiate and spread the synchronized bursting. The changes in timing in the neuronal activation pattern reflecting changes in the leader-follower neurons relationship can thus be used as an indicator for network level potentiation. 


\section{Chapter 4}

\section{Materials and methods}

\subsection{Experimental Preparation}

\subsubsection{Cell culture, transduction and multi-electrode ar- ray recordings}

Hippocampal neurons isolated from E18 Wistar U rats were cultured following primary hippocampal culture procedure from Brewer et al. 1993 and plated on multi-electrode arrays (MEA; type TiN-200-30iR from Multichannel Systems, Reutlingen, Germany) at a density of 1000 cells per $\mathrm{mm}^{2}$. The multi-electrode arrays were coated with $1 \mathrm{ml}$ of a mixture, composed of 600 $\mu \mathrm{l}$ poly-D-lysine $(50 \mu \mathrm{g} / \mathrm{ml})$ and $200 \mu \mathrm{l}(10 \mu \mathrm{g} / \mathrm{ml})$ laminin dissolved in 15 $\mathrm{ml}$ bidistilled water, before plating the cells on it. All animals were kept and bred in the animal house of the Max Planck Institute for Experimental Medicine according to the German guidelines for experimental animals. Animal experiments were carried out with authorization of the responsible federal state authority. The MEAs were covered with the Teflon fluorinated ALA-science caps (ALA scientific instruments, US). The cells were kept in an incubator at $37^{\circ} \mathrm{C}, 8 \% \mathrm{CO} 2$ and $90 \%$ humidity. The cultures were transduced after 14 days after cells plating with AAV-CAG-ChR2-YFP virus (Petreanu et al., 2009, Suska et al., 2013). The titer of the viral particle solution is 
$5^{*} 10^{6}$ t.u. per ul (t.u. $=$ transforming units) which is suitable for cell culture purpose. The transduction efficiency was quantified by counting the number of cells showing YFP fluorescence under epifluorescent microscope (Axiovert 200, Zeiss, Germany). A x20 objective was used showing a 1.1 $\mathrm{mm}^{2}$ field of view in which ratio of transduced neurons to the total number of neurons were counted. The transduction efficiency was consistent among cultures showing an average efficiency varying from 70 to $80 \%$. Recordings were done after 21 days in vitro. The recordings were made on a 60 channel MEA amplifier (MEA-1060 Inv, Multichannel Systems, Reutlingen, Germany). Data from MEAs were captured at $25 \mathrm{kHz}$ using a 64-channel A/D converter and MC_Rack software (Multichannel Systems, Reutlingen, Germany). After high pass filtering (Butterworth 2nd order, $100 \mathrm{~Hz}$ ) action potentials are detected in a cutout recorded $1 \mathrm{~ms}$ before and $2 \mathrm{~ms}$ after crossing a threshold of $-20 \mu \mathrm{V}$, which was $>3$ times standard deviations of the baseline activity.

\subsubsection{Whole field blue light stimulation}

Two protocols of whole field blue light stimulation were used: (1) 40 repetitions of 1 second rectangular (pulsed) light pulses and (2) fade-in stimulation designed as 40 repetitions of slowly ramped light waveform up to the level of constant pulses with frequency of $0.5 \mathrm{~Hz}$.

For the study in Chapter 2, eighteen experiments with pulsed stimulation on eighteen cultures and sixteen experiments with fade-in stimulation, on sixteen cultures, were performed. In each experiment, before the onset of the stimulation, the spontaneous activity of the culture was recorded for 5 minutes. Then the culture was stimulated with one of the two stimulation protocols. After offset of the stimulation the spontaneous activity was recorded for 12 minutes.

For the study in Chapter 3, twelve datasets with pulsed stimulation and twelve datasets with fade-in stimulation are used. 


\subsubsection{Control experiments}

In Chapter 2, two types of control experiments are performed: (1) experiments on transduced cultures without light stimulation (7 experiments on 7 cultures) and (2) experiments on non-transduced cultures stimulated with pulsed light stimulation protocol (5 experiments on 5 cultures).

\subsubsection{Pharmacological experiments}

In Chapter 2, in order to investigate the contribution of different receptors to the observed change in bursting dynamics, experiments were performed under pharmacological synaptic blockade by using pulsed light stimulation protocol (explained in the above section "whole field blue light stimulation"). The following mixtures of synaptic blockers were used: (1) experiments with $100 \mu \mathrm{M}$ APV and $100 \mu \mathrm{M}$ Picrotoxin were used to investigate the AMPA receptor mediated effects (a total of 10 experiments from 10 cultures) and (2) experiments with $50 \mu \mathrm{M}$ NBQX and $100 \mu \mathrm{M}$ Picrotoxin were used to investigate the NMDA receptor mediated effects (a total of 10 experiments from 10 cultures). The blockers were applied to the MEAs prior to the experiment and left to stabilize for a couple of minutes before the recording and photo-stimulation is performed.

\subsection{Network Dynamics Data Analysis}

\subsubsection{Active Electrode (AE) Identification}

Quantification of burst dynamics was restricted to the subset of active electrodes. Active electrodes (AE) were defined as an electrode that has a spontaneous firing rate of more than $0.1 \mathrm{~Hz}$ (Fig. 4.1k). 


\subsubsection{Mean Firing Rate of AEs}

The mean firing rate of the active electrodes was computed as the total number of action potentials recorded by active electrodes divided by the duration of the recording and the number of AEs:

$$
\text { Firing rate of } A E s=\frac{\text { No of action potentials }}{\text { Noof AE } \times \text { Time interval }}
$$

\subsubsection{Peri-stimulus time histogram}

Peri-stimulus time histograms (PSTHs) were calculated using a 20 msec time bin. The level of activity of individual cultures was characterized by the corresponding spontaneous average firing rate, which varies from culture to culture. The average PSTH was obtained from the PSTHs of each experiment normalized with the spontaneous average firing rate before stimulus of the corresponding culture.

\subsubsection{Burst Detection}

For burst detection we have modified the method suggested by Wagenaar et al. (2005a). Bursts were defined as sequences of at least two spikes with all inter-spike intervals lower than a threshold value. The threshold was defined as $1 / 4$ of the inverse average firing rate of all active electrodes (Fig. 4.1c). After detecting bursts on all active electrodes, they were sorted in temporal order. A synchronized burst was defined as a group of bursts across several electrodes that overlapped in time (Fig. 4.1d). After detecting all synchronized bursts, the synchronized bursts that were separated by less than $5 / 4$ of the threshold, inter-spike intervals were merged into one synchronized burst. Normalized average intra-burst firing rate of one experiment before and after fade-in stimulation is shown in Fig. 4.1p,f. In order to obtain the intra-burst firing rate the spike trains of active electrodes were convolved with a Gaussian kernel of standard deviation of $5 \mathrm{~ms}$ and summed up over 
all active electrodes. The average over IBFRs can be done either by aligning the bursts to the onset of the detected bursts (red line) or by aligning them to the first peak (blue line), as reported by e.g. Eytan and Marom (2006). In order to compare the IBFR after stimulus to before stimulus, the average IBFR before and after stimulus is normalized to the peak of average IBFR before stimulus.

Three main quantities are used to characterize the modification of burst structure: (1) firing rate, (2) burst occurrence rate, and (3) intra-burst firing rate. All calculated as a function of time using non-overlapping time bins.

Burst occurrence rate (BR) was defined as the rate of the detected synchronized bursts in a time bin of 10 seconds (Fig. 4.17),

$$
\text { Burst occurance rate }=\frac{\text { No of bursts }}{\text { Time interval }}
$$

The firing rate and burst occurrence rate of each experiment was normalized to the average FR and BR during the spontaneous activity period of the corresponding experiment. The average normalized firing rate and burst occurrence rate over different experiments is the mean value at each time bin over the normalized firing rate and burst occurrence rate of all experiments. Intra-burst firing rate (IBFR) was computed as the total number of action potentials within a synchronized burst (burst size) divided by the burst duration defined as the time interval between the onset and offset of the corresponding synchronized burst (Fig. 4.1d),

$$
\text { Intra - burst firing rate }=\frac{\text { Burst size }}{\text { Burst duration }} \text {. }
$$

In order to compute the average normalized IBFR over all experiments, first the average IBFR of the detected bursts in windows of 10 seconds were computed for each experiment, then the IBFR was normalized to the mean IBFR of the spontaneous activity period of the corresponding experiment and finally the average over all experiments taken.

As mentioned before, there is variation in the level of activity of individual 
cultures, therefore, we computed all of these quantities normalized to the spontaneous activity before stimulus. Averages over all experiments with the same experimental protocol were obtained of these normalized quantities. The mean of these quantities after stimulation, 5 minutes before ending of recording was compared to the mean of the unperturbed spontaneous activity before stimulation in order to test and ensure the reproducibility of the observed effect. The significance of change of the normalized averaged FR, IBFR and BR were assessed using the Wilcoxon rank sum test. This test has the null hypothesis that the two vectors are independent samples from identical continuous distribution with equal medians. The bootstrap 95\% confidence intervals of the means were computed by taking 10000 shuffled random samples from individual experiments.

Silent period is a period of almost no activity after offset of the stimulation. It is defined as the duration between offset of the stimulation and onset of the first synchronize burst after stimulation. As the silent period varies between experiments and also the duration of resuming the ongoing burst activity varies between experiments, in order to compare the change in the activity on average, we took last 5 minutes to compare to spontaneous activity which is the period that all experiments are in the stationary active state again. 


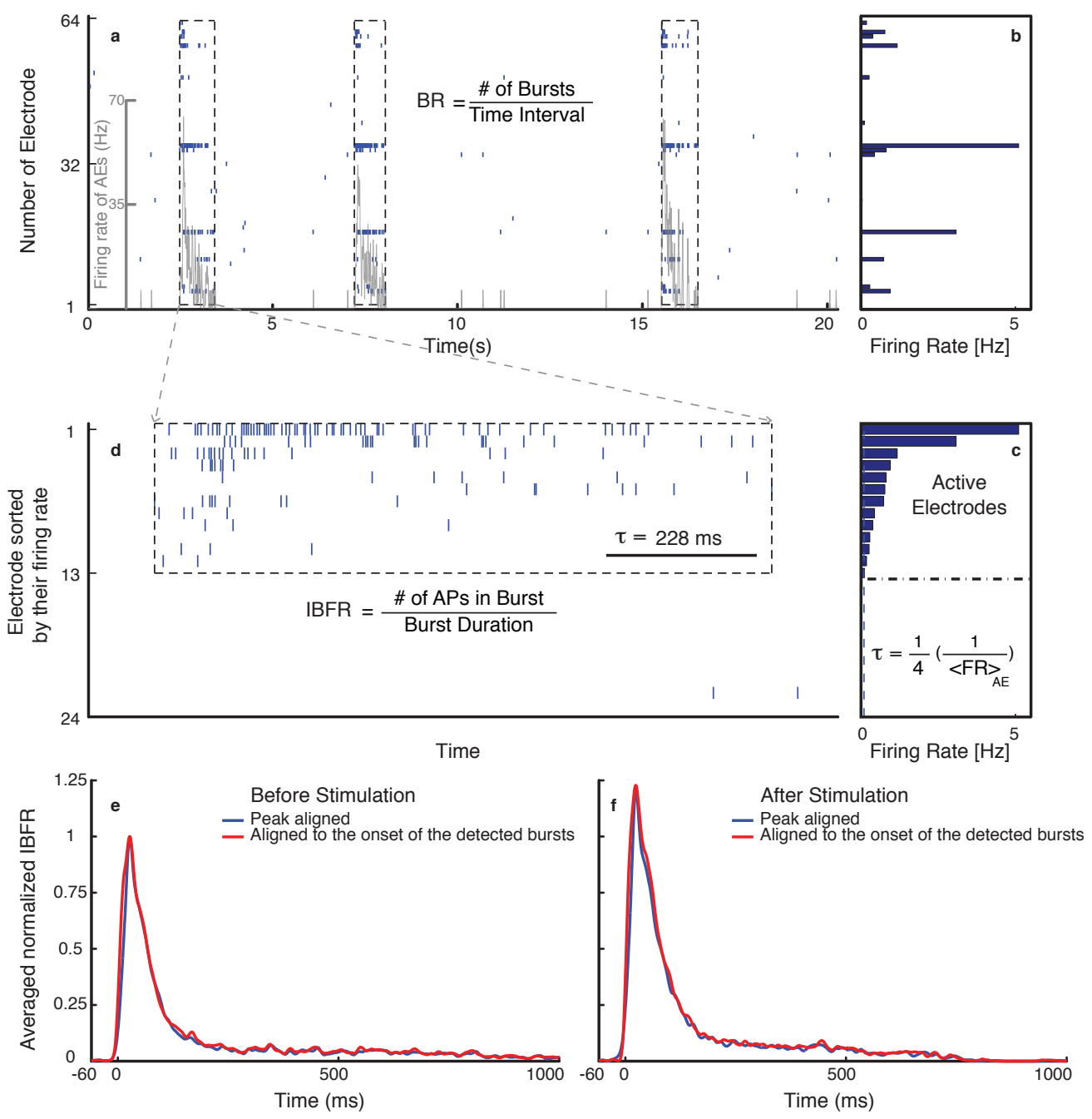

Figure 4.1: Burst detection algorithm. Panel (a) shows typical electrical activity of a culture recorded via all 60 channels and the corresponding firing rate of AEs (gray line). In (b), the average spontaneous firing rates over 300s of the corresponding electrodes are shown. The graph (c) shows the rank ordered firing rates of all electrodes. Selection of the active electrodes (AE), electrodes with average firing rate larger than $0.1 \mathrm{~Hz}$ is illustrated. The threshold for detecting a burst is defined as $1 / 4$ of the inverse average firing rate of all active electrodes. Panel (d) shows the raster plot of an example burst. The threshold ISI, $\tau$, is marked in (d) for comparison. Panels (e,f) show the normalized average IBFR of one experiment with fade-in stimulation before and after stimulus (normalized to the peak of average IBFR before stimulus), by aligning the bursts by the onset of the detected burst (red line) and by aligning bursts by their first peak (blue line). 


\subsubsection{Cross Correlation}

The spike trains of active electrodes were first converted to a binary sequence. The binarized spike train was then convolved with a Gaussian kernel of standard deviation of $5 \mathrm{~ms}$ in order to obtain spike density functions. The spike density functions were then used in the computation of the cross-correlation functions between pairs of electrodes. For each data set, the cross-correlations between all possible combinations of active electrodes pairs were computed. The cross-correlation functions were normalized by the product of the standard deviation of the signals to obtain the cross-correlation coefficients. Then the cross-correlations between all possible pairs of active electrodes were averaged for each data set. Subsequently all data sets were averaged in order to compute the overall average across all data sets. The computation of the average cross-correlation was done for unperturbed spontaneous activity before stimulation (the 5 minutes just before the stimulation) and for after stimulation (the last 5 minutes of the recording). Jackknife confidence intervals were calculated by computing the average cross-correlation function over all experiments removing one electrode at a time. We used a total number of 228 Jackknife samples for the pulsed photo-stimulation condition and 282 Jackknife samples for the fade-in photo-stimulation condition.

\subsection{Leaders and Followers}

\subsubsection{Leader Detection}

In order to detect leader electrodes, different methods have been previously suggested. For example, in the study by Ham et al. (2008), leader electrodes are simply defined as the electrodes that lead at least $4 \%$ of all network bursts. To quantify the leader electrodes, I have modified the method suggested by Eckmann et al. (2008). In this method, it is considered that the leader electrodes are not just electrodes with high activity, which therefore are statistically more often the first ones to fire. To qualify as a leader, 
they require that a trigger's probability to lead bursts should be significantly higher than it's probability to fire in the low-rate quiet intervals (or interburst interval). The rational here is that some electrodes spike only in the burst, but electrodes that are able to be triggers are those which can spike in the quiet phase. They have different definition for burst detection compared to the definition I have proposed and used in this study. Their definition lets them to divide spikes into four classes: in-burst, pre-burst, absorbed burst and isolated, with each spike belonging to one and only one class. Basically, by their definition, a spike is in a burst if it is in a group of many spikes that follow each other closely, which by my definition it is equivalent to be involved in a synchronized network burst. Pre-burst or aborted burst is considered if a spike is in a sequence of spikes that are close enough in time so that communication between them is still possible. The distinction between preburst and aborted burst depends on whether the spike is eventually followed by a synchronized burst or not. In my definition, the spikes in pre-burst are already considered to be in a synchronized burst and aborted bursts are the detected bursts on individual electrodes which don't have temporal overlap with other bursts to be considered as a synchronized burst. Therefore, in comparison to their study, I consider spikes to be either in synchronized burst or in quiet period. Considering this difference in burst definition, I have modified and used the method suggested by Eckmann et al. (2008) as explained below.

Let $\mathrm{M}$ be the total number of detected synchronized bursts. For each $A E_{n}$, $e_{n}$ is defined as the total number of evoked spikes in inter-burst intervals, $q_{n}=\frac{e_{n}}{\sum_{n^{\prime}} e_{n^{\prime}}}$ is the probability of a spike being evoked in inter-burst interval. The probability for $A E_{n}$ to be a spurious or random trigger $\mathrm{F}$ times is given by $p_{n}(F)$ :

$$
\left(\begin{array}{c}
M \\
F
\end{array}\right) q_{n}\left(1-q_{n}\right)^{M-F} .
$$

In the limit of large $\mathrm{M}$ and reasonable $q_{n}$, this distribution is approximated by a Gaussian of mean $M q_{n}$ and variance $M q_{n}\left(1-q_{n}\right)$. On the other hand, 
let's denote $f_{n}$ as the actual number of bursts that $A E_{n}$ leads (note that $\left.\sum_{n} f_{n}=M\right)$.

Thus one can have a score on which to test triggering. Therefore $\alpha_{n}$, called a 'leader score', decide that an $A E_{n}$ is a leader if it scores at least 3SD above the natural expectation value. Therefore the criterion for leadership of $A E_{n}$ is

$$
\alpha_{n}=\frac{f_{n}-M q_{n}}{\sqrt{M q_{n}\left(1-q_{n}\right)}}>3 .
$$

If a leader electrode has leaded less than $3 \%$ of the bursts, it's excluded from being a leader.

As it is known, recording by MEA can record from only $0.1 \%$ of the total population of neurons in the culture and as mentioned before, the spike detection is done by threshold crossing. Therefore, if in a very rare case, we have detected $q_{n}=0$, we have considered that there might have been at least one spike which has been evoked but it has not been detected in the recording, so, instead of $q_{n}=0$, we have considered $q_{n}=\frac{1}{\sum_{n^{\prime}} e_{n^{\prime}}}$.

Besides the detected leader electrodes before and after stimulus, the rest of the electrodes are denoted as followers.

\subsubsection{Intra-burst firing rate (IBFR)}

In order to obtain the IBFR, the spike trains of AEs for each synchronized burst were convolved with a Gaussian kernel of standard deviation of $5 \mathrm{~ms}$ and averaged over all bursts at each AE.

\subsubsection{Peak Delay}

Peak delay is defined as the time delay of the first peak which is larger than $2 / 3$ of maximum peak of IBFR at each electrode from onset of the synchronized burst. 


\section{Chapter 5}

\section{General discussion}

In the present work, a way to enhance the collective dynamics of cultured hippocampal neurons was presented. This study was done based on the underlying characteristic behavior of in-vitro neuronal cultures, such as bursting and the hierarchical activation order within such synchronized bursting activities. The optical network electrophysiology designed by A. El Hady, enabled us to do this study by using the combination of non-invasive optical stimulation using optogenetic tools together with extracellular recording using multi-electrode arrays.

In this study, the combination of electrical recoding using multi-electrode array with optical stimulation of cultured Channelrhodopsin-2 transducted hippocampal neurons was used to study and manipulate network burst synchronization. We found low frequency photo-stimulation protocols that are sufficient to induce potentiation of network bursting, modifying bursting dynamics and increasing interneuronal synchronization. This study is the first report of network level potentiation using optical stimulation. Previous studies of plasticity in neuronal cultures have primarily use electrical or chemical induction methods (Maeda et al., 1998; Ivenshitz and Segal, 2006). Surprisingly, slowly fading-in light stimulation, which substantially delayed and reduced light driven spiking, was at least as effective in reorganizing network dynamics as much stronger pulsed light stimulation. Our study shows that mild stimulation protocols that do not enforce particular activity patterns 
onto the network can be highly effective inducers of network-level plasticity. In the first part of the work, the overall character of network reorganization was examined by using the set of firing statistics consisting of: firing rate, intra-burst firing rate and burst occurrence rate. After offset of the stimulation, both the mean firing rate and intra-burst firing rate increased significantly compared to the spontaneous activity of the culture as a result of network-level potentiation. The intra-burst firing rate increased with the same magnitude as the firing rate. Due to the fact that the majority of the spikes occur within the bursts, the increase firing rate is mainly responsible for increased intra-burst firing rate. The burst occurrence rate also increased significantly after offset of the stimulation compared to the unperturbed spontaneous activity, which is also reflected as a decrease in inter-burst interval. These changes in the collective network dynamics indicate a specific increase of network excitability due to enhanced excitatory interactions. These results are consistent with findings from previous studies. For instance, Maeda et al. (1998) were able to induce an increase of the intraburst firing rate and the burst occurrence rate using high frequency tetanic stimulation. However, using high frequency stimulation might exhaust the network. Thus, our stimulation protocol has the advantage of inducing lasting potentiation even without a need for high frequency stimulation.

We also examined changes in correlation structure of the network after offset of the stimulation. The results shows an increase in the amplitude of cross-correlation functions after offset of the stimulation, which reflects an increase in spike synchronization. The increase in spike synchronization suggests that there is a tighter coupling between neurons rather than a change in the overall activity level of burst firing. The width of the cross-correlation functions was generally much smaller than the mean burst duration either before or after stimulation for both pulsed and fade-in photo-stimulation. This demonstrates that the change in correlation structure results from modifications in the fine structure within the burst. Interestingly, the enhancement of the average cross-correlation was more pronounced in the case of fadein stimulation than in the case of pulsed stimulation, which highlights the 
effectiveness of mild fade-in photo-stimulation.

The pharmacological analyses indicate that the observed network-level potentiation is mediated via a mixed mechanism involving both NMDA and AMPA receptors.

The synchronized burst initiation involves two distinct processes: the ignition of the burst via one of the so called "leader neurons" and the recruitment of "follower" neurons which induce the propagation of synchronized burst. As previously mentioned, using low frequency global photo-stimulaiton was sufficient to modify intra-burst firing rate dynamics. Therefore, it was interesting to study if this change can also influence the leader-follower relationships. To do this, the electrodes were divided into two subsets of leaders and followers. The leader neurons were mainly robust and did not change upon stimulation. The maximum peak of intra-burst firing rate of leader and follower electrodes increases significantly after stimulation for both fade-in and pulsed stimulation. Interestingly this increase is larger in case of pulsed stimulation than in the case of fade-in stimulation. On the other hand, after the fade-in stimulation the intra-burst firing rate peak delay of follower neurons gets shorter compared to unperturbed spontaneous activity, reflecting that the follower neurons fire much faster in relation to the leader neurons. This means that fade-in stimulation brings the follower neurons into a tighter relationship with the leader neurons.

In this study, we presented two simple photo-stimulation protocols able to modify the intrinsic collective dynamics of cultured neuronal networks. These two photo-stimulation protocols are designed as 40 repetitions of 1 s rectangular (pulsed) or slowly ramped light waveform (fade-in) up to the level of constant pulses with frequency of $0.5 \mathrm{~Hz}$. Although, neither of these two protocols enforce particular activity patterns onto the network, it is shown that they can effectively induce the network-level plasticity. Moreover, the leaderfollower analysis shows that the initiation and propagation dynamics of synchronized bursts are differentially modulated by different photo-stimulation paradigms. Intriguingly, our results indicated that slowly fading-in light stimulation, can be even more effective in potentiating network-level plas- 
ticity which is reflected in a tighter leader-follower neurons relationship and faster recruitment of follower neurons after initiation of the burst via one of the leader neurons. The PSTH analysis shows that the induced photoresponse activity is quite different between pulsed and fade-in stimulation. The pulsed stimulation rapidly trigger a highly synchronized activation of the network, whereas in case of fade-in stimulation the firing rate rose much more slowly and reach a maximum firing rate around 1.5 fold lower than in case of the pulsed stimulation. Therefore, from the PSTH analysis, it is clear that the pulsed stimulation makes neurons to respond to the light in much more tightly synchronized manner compared to fade-in stimulation. A relevant question in this respect is why the pulsed stimulation is less effective despite it's ability to activate the network with even higher firing rates and also much more synchronized compared to fade-in stimulation. In case of pulsed stimulation, it seems that the network is overrode and the activation is completely imposed from the outside and has not much to do with the preexisting structure of the network. However, one can hypothesize that fade-in stimulation is more effective because it might induce the patterns which are more similar to the intrinsic patterns of activity that are shaped by the real connectivity of the network. With fade-in stimulation, it might be possible to trigger those neurons that would start a burst spontaneously and help them to initiate a synchronized burst, which means it triggers activity patterns that are structured by the connectivity of the network and therefore enhance the activity more effectively. Precise spike timing is known to be essential for many forms of synaptic plasticity (Caporale and Dan, 2008). STDP theory tells us that the first spike matters. Moreover, the time delay between pair activations is also playing an important role. Therefore, the tightening of the leader-follower relationship might be due to synaptic strengthening in a similar manner as in spike-timing dependent plasticity (Dan and Poo 2004) owing to the enhancement of inter-neuronal correlations during fade-in stimulation or due to the increased number of spikes within the bursts as a result of photo-stimulation. For further investigating this hypothesis, I suggest to use stimuli of different fade-in slopes, both larger and smaller than the slope used in this study. More in detail, I propose to keep the same maximum 
intensity of light and same stimulus duration and systematically vary the ramp slope. I would expect to observe an stronger effect as the ramped light waveform is slowed down - potentially for shallow slopes even stronger than in the current results. 


\section{Bibliography}

L. F. Abbott and S. B. Nelson. Synaptic plasticity: taming the beast. Nat. Neurosci. Suppl. 3, 1178-1183 (2000)

L. F. Abbott, W. G. Regehr. Synaptic computation. Nature 431: 796-803 (2004)

W. Abraham, B. Gustafsson, H. Wigström. Long-term potentiation involves enhanced synaptic excitation relative to synaptic inhibition in guinea-pig hippocampus. J. Physiol. 394:367 -380 (1987)

C. D. Aizenman, D. J. Linden. Rapid, synaptically driven increases in the intrinsic excitability of cerebellar deep nuclear neurons. Nat. Neurosci. 3:109$111(2000)$

A. Arai, G. Lynch. AMPA receptor desensitization modulates synaptic responses induced by repetitive afferent stimulation in hippocampal slices. Brain Res 799: 235-242 (1998)

A. Bacci, C. Verderio, E. Pravettoni, M. Matteoli. Synaptic and intrinsic mechanisms shape synchronous bursts in hippocampal neurons in culture. Eur. J. Neurosci. 11, 389-397 (1999)

D. J. Bakkum, Z. C. Chao, S. M. Potter. Spatio-temporal electrical stimuli shape behavior of an embodied cortical network in a goal-directed learning task. J Neural Eng 5:310-323 (2008a)

D. J. Bakkum, Z. C. Chao, S. M. Potter. Long-term activity-dependent plasticity of action potential propagation delay and amplitude in cortical networks. PloS One 3:e2088 (2008b) 
D. Battaglia, A. Witt, F. Wolf, T. Geisel. Dynamic Effective Connectivity of Inter-Areal Brain Circuits. PLoS Comput Biol 8(3), e1002438 (2012)

J. M. Beggs and D. Plenz. Neuronal Avalanches in Neocortical Circuits. J. Neurosci. 23(35):11167-11177 (2003)

G. Q. Bi and M. M. Poo. Synaptic modifications in cultured hippocampal neurons: dependence on spike timing, synaptic strength, and postsynaptic cell type. J. Neurosci. 18, 10464-10472 (1998)

T. V. P. Bliss, A. R. Gardner-Medwin. Long-lasting potentiation of synaptic transmission in the dentate area of the unanaesthetized rabbit following stimulation of the perforant path. J. Physiol. 232:357-74 (1973)

T. V. P. Bliss, T. Lømo. Long-lasting potentiation of synaptic transmission in the dentate area of the anaesthetized rabbit following stimulation of the perforent path. J. Physiol. 232:331 -356 (1973)

F. Blumhagen, P. Zhu, J. Shum, Y. P. Schärer, E. Yaksi, K. Deisseroth, R. W. Friedrich. Neuronal filtering of multiplexed odour representations. Nature 479(7374), 493-8 (2011)

Y. Bobkov, I. Park, K. Ukhanov, J. Principe, B. Ache. Cellular basis for response diversity in the olfactory periphery. PLoS One. 7(4):e34843 (2012)

L. L. Bologna, T. Nieus, M. Tedesco, M. Chiappalone, F. Benfenati, S. Martinoia. Low-frequency stimulation enhances burst activity in cortical cultures during development. Neuroscience 165(3), 692-704 (2010)

E. Boyden, F. Zhang, E. Bamberg, G. Nagel \& K. Deisseroth. Millisecondtimescale, genetically targeted optical control of neural activity. Nat. Neurosci. 8, 1263-1268 (2005)

G. J. Brewer, M. D. Boehler, A. N. Ide, B. C. Wheeler. Chronic electrical stimulation of cultured hippocampal networks increases spontaneous spike rates. Journal of Neuroscience Methods 184, 104-109 (2009)

G. J. Brewer, J. R. Torricelli, E. K. Evege, and P. J. Price. Optimized survival of hippocampal neurons in B27-supplemented Neurobasal, a new serum-free medium combination. J. Neurosci. Res. 35, 567-576 (1993) 
R. E. Brown \& P. M. Milner. The legacy of Donald O. Hebb: more than the Hebb synapse. Nature Rev. Neurosci. 4, 1013-1019 (2003)

G. Buzsáki. Rhythms of the Brain. Oxford University Press. (2006)

A. R. Cantrell, W. A. Catterall. Neuromodulation of Na + channels: An unexpected form of cellular plasticity. Nat. Rev. Neurosci. 2: 397-407 (2001)

N. Caporale, Y. Dan. Spike timing-dependent plasticity: a Hebbian learning rule. Annual Review of Neuroscience. 31:25-46 (2008)

L. Cathala, D. Paupardin-Tritsch. Neurotensin inhibition of the hyperpolarizationactivated cation current $\left(\mathrm{I}_{\mathrm{h}}\right)$ in the rat susbstantia nigra pars compacta implicates the protein kinase C pathway. J. Physiol. 503: 87-97 (1997)

M. Chiappalone, P. Massobrio, S. Martinoia. Network plasticity in cortical assemblies. Eur J Neurosci. 28, 221-237 (2008)

E. Cohen, M. Ivenshitz, V. Amor-Baroukh, V. Greenberger, M. Segal M. Determinants of spontaneous activity in networks of cultured hippocampus. Brain Research 1235: 21-30 (2008)

D. Cohen, M. Segal. Homeostatic presynaptic suppression of neuronal network bursts. J Neurophysiol 101: 2077-2088 (2009)

D. Cohen, M. Segal. Network bursts in hippocampal microcultures are terminated by exhaustion of vesicle pools. J Neurophysiol 106: 2314-2321 (2011)

V. Crépel, D. Aronov, I. Jorquera, A. Represa, Y. Ben-Ari, R. Cossart. A parturition-associated nonsynaptic coherent activity pattern in the developing hippocampus. Neuron 54, 105-120 (2007)

A. Czarnecki, A. Tscherter, J. Streit. Network activity and spike discharge oscillations in cortical slice cultures from neonatal rat. European Journal of Neuroscience 35:375 - 388 (2012)

Y. Dan, M. Poo. Spike Timing-Dependent Plasticity: From Synapse to Perception. Physiol Rev 86: 1033-1048 (2006)

G. Daoudal, Y. Hanada, D. Debanne. Bi-directional plasticity of EPSP-spike 
coupling in CA1 hippocampal pyramidal neurons. Proc. Natl. Acad. Sci. 99:14512 -14517 (2002)

P. Darbon, L. Scicluna, A. Tscherter, J. Streit. Mechanisms controlling bursting activity induced by disinhibition in spinal cord networks. Eur J Neurosci 15: 671-683 (2002)

B. Dargent, F. Julien, F. Couraud. Internalization of voltage-dependent sodium channels in fetal rat brains: A study of the regulation of endocytosis. J. Neurochem. 65:407-413 (1995)

D. Debanne, B. H. Gahwiler, S. M. Thompson. Long-term synaptic plasticity between pairs of individual CA3 pyramidal cells in rat hippocampal slice cultures. J. Physiol. 507(Pt. 1):237-47 (1998)

M. Dranias, H. Ju, E. Rajaram, A. VanDongen. Short term memory in network of dissociated cortical neurons. J Neurosci. 33(5):1940-1953 (2013)

J. P. Eckmann, O. Feinerman, L. Gruendlinger, E. Moses, J. Soriano, T. Tlusty. The physics of living neural networks. Physics Reports 449: 54-76 (2007)

J. P. Eckmann, S. Jacobi, S. Marom, E. Moses, C. Zbinden. Leader neurons in population bursts of $2 \mathrm{D}$ living neuronal networks. New Journal of Physics 10: $015011(2008)$

U. Egert, Th. Knott, C. Schwarz, M. Nawrot, A. Brandt, S. Rotter, M. Diesmann. MEA-Tools: an open source toolbox for the analysis of multielectrodedata with Matlab. J. Neurosci. Meth. 117: 33-42 (2002)

A. El Hady*, G. Afshar*, K. Bröking, O. M. Schlüter, T. Geisel, W. Stühmer and F. Wolf. Optogenetic stimulation effectively enhances intrinsically generated network synchrony. Front. Neural Circuits 7:167. doi: 10.3389/fncir.2013.00167 (2013)

D. Eytan, N. Brenner, S. Marom. Selective adaptation in networks of cortical neurons. J. Neurosci. 23(28):9349-56 (2003)

D. Eytan, S. Marom. Dynamics and effective topology underlying synchronization in networks of cortical neurons 26(33): 8465 - 8476 (2006) 
O. Feinerman and E. Moses. Transport of Information along Unidimensional Layered Networks of Dissociated Hippocampal Neurons and Implications for Rate Coding. J. Neurosci. 26(17):4526-4534 (2006)

O. Feinerman, A. Rotem, E. Moses. Reliable neuronal logic devices from patterned hippocampal cultures. Nature Physics 4(12), 967-73 (2008)

O. Feinerman, M. Segal and E. Moses. Signal propagation along unidimensional neuronal networks. J. Neurophysiol. 94, 3406 - 16 (2005)

O. Feinerman, M. Segal and E. Moses. Identification and dynamics of spontaneous burst initiation zones in unidimensional neuronal cultures. Journal of Neurophysiology, 97(4), 2937-2948 (2007)

K. Ganguly, L. Kiss, M. M. Poo. Enhancement of presynaptic neuronal excitability by correlated presynaptic and postsynaptic spiking. Nat. Neurosci. 3:1018-1026 (2000)

K. R. Gee, K. A. Brown, W. N. Chen, J. Bishop-Stewart, D. Gray, I. Johnson. Chemical and physiological characterization of fluo- $4 \mathrm{Ca}(2+)$-indicator dyes. Cell Calcium 27(2):97-106 (2000)

C. S. Goodman, C. J. Shatz. Developmental mechanisms that generate precise patterns of neuronal connectivity. Cell 72 (Suppl), 77-98 (1993)

G. W. Gross. Simultaneous single unit recording in-vitro with a photoetched laser deinsulated gold multimicroelectrode surface. IEEE Trans. Biomed. Eng. 26(5):273-9 (1979)

G. W. Gross, B. K. Rhoades, D. L. Reust, F. U. Schwalm. Stimulation of monolayer networks in culture through thin-film indium-tin oxide recording electrodes. J. Neurosci. Methods 50(2):131-43 (1993)

L. A. Gunaydin, O. Yizhar, A. Berndt, V.S. Sohal, K. Deisseroth, P. Hegemann. Ultrafast optogenetic control. Nat Neurosci 13, 387-392 (2010)

M. I. Ham, L. M. A. Bettencourt, F. D. McDaniel, G. W. Gross. Spontaneous coordinated activity in cultured networks: analysis of multiple ignition sites, primary circuits, and burst phase delay distributions. J. Comput. Neurosci. 24: 346-357 (2008) 
X. Han, X. Qian, P. Stern, A. S. Chuong, E. S. Boyden. Informational lesions: optical perturbation of spike timing and neural synchrony via microbial opsin gene fusions. Front Mol Neurosci. 2, 12 (2009)

R. G. Harrison. The outgrowth of the nerve fiber as a mode of protoplasmic movement. J Exp Zool. 9(4):787-846 (1910)

D. Hebb. The Organization of Behavior: A Neuropsychological Theory,Wiley, NewYork (1949)

G. Heimer, M. Rivlin, Z. Israel, H. Bergman. Synchronizing activity of basal ganglia and pathophysiology of Parkinson's disease. J Neural Transm Suppl. 70, 17-20 (2006)

S. Herzig, J. Neumann. Effects of serine/threonine protein phosphatases on ion channels in excitable membranes. Physiol. Rev. 80:173 -210 (2000)

M. Holtkamp, K. Buchheim, M. Elsner, J. Matzen, F. Weissinger, H. Meierkord. Status epilepticus induces increasing neuronal excitability and hypersynchrony as revealed by optical imaging. Neurobiol. Dis. Jul. 43(1): 220-7 (2011)

K. Hu, S. Huang, Y. N. Jan, L. Y. Jan. ATP-sensitive potassium channel traffic regulation by adenosine and protein kinase C. Neuron 38:417 -432 (2003)

A. N. Ide, A. Andruska, M. Boehler, B. C. Wheeler, G. J. Brewer. Chronic network stimulation enhances evoked action potentials. Journal of Neural Engineering 7(1): 16008 (2010)

M. Ivenshitz, M. Segal. Simultaneous NMDA-dependent long-term potentiation of EPSCs and long-term depression of IPSCs in cultured rat hippocampal neurons. J Neurosci. 26(4),1199-210 (2006)

J. M. Jester, L. W. Campbell, T. J. Sejnowski. Associated EPSP-spike potentiation induced by pairing orthodromic and antidromic stimulation in rat hippocampal slices. J. Physiol. 484:689 -765 (1995)

Y. Jimbo, T. Tateno, H. P. C. Robinson. Simultaneous induction of pathwayspecific potentiation and depression in networks of cortical neurons. Biophys. J. 76670 (1999) 
J. Jones, E. A. Stubblefield, T. A. Benke, K. J. Staley. Desynchronization of glutamate release prolongs synchronous CA3 network activity. J Neurophysiol 97: 3812-3818 (2007)

E. Kandel, W. A. Spencer. Electrophysiology of hippocampal neurons. II. After-potentials and repetitive firing. J Neurophysiol 24, 243-59 (1961)

A. Kepecs, J. Lisman. Information encoding and computation with spikes and bursts. Network 14(1), 103-18 (2000)

R. Krahe, F. Gabbiani. Burst firing in sensory systems. Nat Rev Neurosci. 5(1), 13-23 (2004)

P. Kruskal, L. Li, J. MacLean. Circuit reactivation dynamically regulates synaptic plasticity in neocortex. Nature Communications 4. doi:10.1038/ncomms3574 (2013)

J. le Feber, J. Stegenga, W. L. C. Rutten. The Effect of Slow Electrical Stimuli to Achieve Learning in Cultured Networks of Rat Cortical Neurons. PLoS One. 5(1) :e8871 (2010)

X. Leinekugel, R. Khazipov, R. Cannon, H. Hirase, Y. Ben-Ari, G. Buzsaki. Correlated bursts of activity in the neonatal hippocampus in-vivo. Science 296(5575):2049-2052 (2002)

N. A. Lesica, G. B. Stanley. Encoding of natural scene movies by tonic and burst spikes in the lateral geniculate nucleus. J Neurosci. 24(47), 10731-40 (2004)

W. B. Levy, O. Steward. Temporal contiguity requirements for long-term associative potentiation/ depression in the hippocampus. Neuroscience 8:791-97 (1983)

Y. Li, W. Zhou, X. Li, S. Zeng \& Q. Luo. Dynamics of learning in cultured neuronal networks with antagonists of glutamate receptors. Biophysical journal, 93(12), 4151-8 (2007)

J. Lisman. The CaM kinase II hypothesis for the storage of synaptic memory.Trends Neurosci. 17(10), 406-12 (1994) 
M. A. Lynch. Long-term potentiation and memory. Physiol. Rev. 84, 87-136 (2004)

E. Maeda, Y. Kuroda, H. P. C. Robinson, A. Kawana. Modification of parallel activity elicited by propagating bursts in developing networks of rat cortical neurones. Eur. J. Neurosci. 10488 (1998)

E. Maeda, H. Robinson, A. Kawana. The mechanisms of generation and propagation of synchronized bursting in developing networks of cortical neurons. J. Neurosci. 15: 6834-6845 (1995)

H. Markram, J. Lübke, M. Frotscher, B. Sakmann. Regulation of synaptic efficacy by coincidence of postsynaptic APs and EPSPs. Science 275, 213-215 (1997)

S. Marom, G. Shahaf. Development, learning and memory in large random networks of cortical neurons: lessons beyond anatomy. Q. Rev. Biophys. 35 $63(2002)$

S. Martinez-Conde, S. L. Macknik, D.H. Hubel. Microsaccadic eye movements and firing of single cells in the striate cortex of macaque monkeys. Nat Neurosci. 3(3), 251-8 (2000)

A. Mazzoni, F. D. Broccard, E. Garcia-Perez, P. Bonifazi, M. E. Ruaro, V. Torre. On the dynamics of the spontaneous activity in neuronal networks. PLoS One. 2(5):e439 (2007)

R. Meier, U. Egert, A. Aertsen, M. Nawrot. FIND - a unified framework for neural data analysis. In press Neural Networks [pdf] (2008)

L. Menendez de la Prida, G. Huberfeld, I. Cohen, R. Miles. Threshold behavior in the initiation of hippocampal population bursts. Neuron 49: 131-142 (2006)

R. Miles, R. D. Traub, R. K. Wong. Spread of synchronous firing in longitudinal slices from the CA3 region of the hippocampus. J Neurophysiol. 60(4), 1481-96 (1988)

M. Minlebaev, Y. Ben-Ari, R. Khazipov. NMDA receptors pattern early activity in the developing barrel cortex in vivo. Cereb Cortex. 19(3), 688-96 (2009) 
E. Molnár. Long-term potentiation in cultured hippocampal neurons. Semin Cell Dev Biol. 766 22(5), 506-13 (2011)

G. Nagel, D. Ollig, M. Fuhrmann, S. Kateriya, A. M. Musti, E. Bamberg, P. Hegemann. Channelrhodopsin-1: a light-gated proton channel in green algae. Science 296:2395-2398 (2002)

G. Nagel, T. Szellas, W. Huhn, S. Kateriya, N. Adeishvili, P. Berthold, D. Ollig, P. Hegemann and E. Bamberg. Channelrhodopsin-2, a directly lightgated cation-selective membrane channel. Proc. Natl. Acad. Sci. USA 100, 13940-13945 (2003)

Y. Nam, J. Chang, D. Khatami, G. J. Brewer, B. C. Wheeler. Patterning to enhance activity of cultured neuronal networks. IEE Proc Nanobiotechnol 151: 109-115 (2004)

E. Neher. Nobel lecture. Ion channels for communication between and within cells. Neuron 8(4):605-12 (1992)

S. Ostojic, N. Brunel, V. Hakim. How connectivity, background activity, and synaptic properties shape the cross-correlation between spike trains. J Neurosci 29, 10234-10253 (2009)

V. Pasquale, S. Martinia, M. Chiappalone. A self-adapting approach for the detection of bursts and network bursts in neuronal cultures. J Comput Neurosci 29:213-229 (2010)

L. Petreanu, T. Mao, S. M. Sternson and K. Svoboda. The subcellular organization of neocortical excitatory connections. Nature 457 (7233),11421145 (2009)

S. M. Potter, D. A. Wagenaar, T. B. DeMarse, in: M. Taketani, M. Baudry (Eds.), Advances in Network Eletrophysiology Using Multi-Electrode Arrays, Springer, NewYork, pp. 215-242 (2006)

P. P. Quilichini, N. Le Van Quyen, A. Ivanov, D. A. Turner, A. Carabalona, H. Gozlan, M. Esclapez, C. Bernard. Hub GABA neurons mediate Gammafrequency oscillations at ictal-like event onset in the immature hippocampus. Neuron, 74: 57 - 64 (2012) 
M. Raghavan, B. Amrutur, K. V. Srinivas and S. K. Sikdar. A study of epileptogenic network structures in rat hippocampal cultures using first spike latencies during synchronization events. Phys. Biol. 9056002 (2012)

J. M. Rebesco, I. H. Stevenson, K. P. Körding, S. A. Solla, L. E. Miller. Rewiring neural interactions by micro-stimulation. Front Syst Neurosci. 4. pii: 39. doi: 10.3389/fnsys.2010.00039 (2010)

A. Reiher, S. Günther, A. Krtschil, H.Witte, A. Krost, T. Opitz, A. de Lima, T. Voigt. in-vitro stimulation of neurons by a planar $\mathrm{Ti}-\mathrm{Au}$-electrode interface. Appl. Phys. Lett. 86, 103901 (2005)

P. Reinagel, D. Godwin, S. M. Sherman, C. Koch. Encoding of visual information by LGN bursts. J Neurophysiol. 5, 2558-69 (1999)

N. L. Rochefort, O. Garaschuk, R. I. Milos, M. Narushima, N. Marandi, B. Pichler, Y. Kovalchuk, A. Konnerth. Sparsification of neuronal activity in the visual cortex at eye-opening. Proc Natl Acad Sci U S A. 106 (35), 15049-54 (2009)

G. Shahaf, S. Marom. Learning in networks of cortical neurons. J. Neurosci. 21 (22):8782-8788 (2001)

L. A. Schrader, A. E. Anderson, A. W. Varga, M. Levy, J. D. Sweatt. The other half of Hebb. Mol. Neurobiol. 25:33 -48 (2002)

L. R. Silva, Y. Amitai, B. W. Connors. Intrinsic oscillations of neocortex generated by layer 5 pyramidal neurons. Science 251(4992), 432-5 (1991)

A. Suska, B. R. Lee, Y. H. Huang, Y. Dong, O. M. Schlüter. Selective presynaptic enhancement of the prefrontal cortex to nucleus accumbens pathway by cocaine. PNAS 110 (2):713-718 (2013)

H. Takahashi, T. Sakurai, H. Sakai, D. Bakkum, J. Suzurikawa, R. Kanzaki. Light addressed single neuron stimulation in dissociated neuronal cultures with sparse expression of ChR2. Biosystems 107(2): 106 - 112 (2012)

M. Tanemoto, A. Fujita, K. Higashi, Y. Kurachi. PSD-95 mediates formation of a functional homeric Kir 5.1 channel in the brain. Neuron 34:387 -397 (2002) 
T. Tchumatchenko, T. Geisel, M. Volgushev, F. Wolf. Signatures of synchrony in pairwise count correlations. Front. Comput. Neurosci. 4,1 (2010) J. Tønnesen, A. T. Sørensen, K. Deisseroth, C. Lundberg, M. Kokaia. Optogenetic control of epileptiform activity. Proc Natl Acad Sci U S A. 106(29), 12162-7 (2009)

M. Tsodyks, T. Kenet, A. Grinvald, A. Arieli. Linking spontaneous activity of single cortical neurons and the underlying functional architecture. Science 286:1943-1946 (1999)

H. Tsubokawa, S. Offermanns, M. Simon, M. Kano. Calcium-dependent persistent facilitation of spike backpropagation in the CA1 area pyramidal neurons. J. Neurosci. 20:4878 -4884 (2000)

G. G. Turrigiano \& S. B. Nelson. Homeostatic plasticity in the developing nervous system. Nature Rev. Neurosci. 5, 97-107 (2004)

W. van Drongelen, H. Koch, C. Marcuccilli, F. Pena, J. M. Ramirez. Synchrony levels during evoked seizure-like bursts in mouse neocortical slices. J Neurophysiol. 90(3):1571-80 (2003)

J. van Pelt, P.S. Wolters, M. A. Corner, W. L. Rutten, G. J. Ramakers. Longterm characterization of firing dynamics of spontaneous bursts in cultured neural networks. IEEE Trans Biomed Eng 51:2051-2062 (2004)

J. van Pelt, I. Vajda, P. S. Wolters, M. A. Corner and G. J. Ramakers. Dynamics and plasticity in developing neuronal networks in vitro Prog. Brain. Res. 147 173-88 (2005)

D. A. Wagenaar, S. M. Potter. Real-time multi-channel stimulus artifact suppression by local curve fitting. J Neurosci Methods. 120(2), 113-20 (2002) D. A. Wagenaar, T. B. DeMarse, S. M. Potter. MEABench: A toolset for multi-electrode data acquisition and on-line analysis. Proc 2nd Intl IEEE EMBS Conf on Neural Eng 518-521 (2005a)

D. A. Wagenaar, R. Madhavan, J. Pine, S. M. Potter. Controlling bursting in cortical cultures with closed-loop multi-electrode stimulation. J Neurosci. 25(3), 680-8 (2005b) 
D. A. Wagenaar, J. Pine, S. Potter. An extremely rich repertoire of bursting patterns during the development of cortical cultures. BMC Neurosci. 7:11. doi: 10.1186/1471-2202-7-11 (2006a)

D. A. Wagenaar, Z. Nadasdy, S. M. Potter. Persistent dynamic attractors in activity patterns of cultured neuronal networks. Physical Rev. E 73, 051907 (2006b)

S. Wang, S. Szobota, Y. Wang, M. Volgraf, Z. Liu, C. Sun, D. Trauner, E. Y. Isacoff and X. Zhang. All optical interface for parallel, remote, and spatiotempoeral control of neuronal activity. Nano Lett. 7, 3859-63 (2007)

Z. Wang, N. I. Xu, C. P. Wu, S. Duan, M. M. Poo. Bidirectional changes in spatial dendritic integration accompanying long-term synaptic modifications. Neuron 37:463 -472 (2003)

M. Weliky. Recording and manipulating the in vivo correlational structure of neuronal activity during visual cortical development. J Neurobiol. 41(1), 25-32 (1999)

H. Yang, W. L. Shew, R. Roy and D. Plenz. Maximal Variability of Phase Synchrony in Cortical Networks with Neuronal Avalanches. J. Neurosci. 32(3):1061-1072 (2012)

O. Yizhar, L. E. Fenno, M. Prigge, F. Schneider, T. J. Davidson, D. J. O'Shea, V. S. Sohal, I. Goshen, J. Finkelstein, J. T. Paz, K. Stehfest, R. Fudim, C. Ramakrishnan, J. R. Huguenard, P. Hegemann, K. Deisseroth. Neocortical excitation/ inhibition balance in information processing and social dysfunction. Nature 477:171-178 (2011)

Y. P. Zhang, N. Holbro, T. G. Oertner. Optical induction of plasticity at single synapses reveals input-specific accumulation of alphaCaMKII. Proc Natl Acad Sci U S A. 105(33), 12039-44 (2008)

L. I. Zhang, H. W. Tao, C. E. Holt, W. A. Harris, M. Poo. A critical window for cooperation and competition among developing retinotectal synapses. Nature 395:37-44 (1998)

R. S. Zucker, W. G. Regehr. Short-term synaptic plasticity. Annu. Rev. Physiol. 64:355-405 (2002) 
P. J. Uhlhaas, W. Singer. Neural synchrony in brain disorders: relevance for cognitive dysfunctions and pathophysiology. Neuron 52:155-168 (2006)

P. J. Uhlhaas, W. Singer. Abnormal neural oscillations and synchrony in schizophrenia. Nat Rev Neurosci. Feb;11(2):100-13 (2010) 